\title{
Supporting Information for: \\ Porphyrin Dyads Bearing Carbon Tethers for Studies of High-Density Molecular Charge Storage on Silicon Surfaces
}

\author{
Patchanita Thamyongkit, Lianhe Yu, Kisari Padmaja, Jieying Jiao, \\ David F. Bocian, and Jonathan S. Lindsey
}

Table of Contents

$\begin{array}{lll}\text { I. } & \text { General Experimental Section } & \text { S1 } \\ \text { II. } & \text { Diacylation Studies } & \text { S1-S2 } \\ \text { III. } & \text { Characterization Data for Selected Compounds } & \text { S3-S29 }\end{array}$

\section{General Experimental Section.}

All ${ }^{1} \mathrm{H}$ NMR (400 MHz) and ${ }^{13} \mathrm{C}$ NMR $(100 \mathrm{MHz})$ were collected in $\mathrm{CDCl}_{3}$ unless noted otherwise. Absorption and fluorescence spectra were collected in toluene at room temperature unless noted otherwise. Mass spectra of porphyrins were obtained via laser desorption mass spectrometry (LD-MS) without a matrix, ${ }^{15}$ laser desorption mass spectrometry with a matrix of POPOP (MALDI-MS), and by high-resolution fast atom bombardment mass spectrometry (FABMS) using a matrix of nitrobenzyl alcohol and polyethylene glycol. Analytical SEC ${ }^{16}\left(\lambda_{\operatorname{det}} 420\right.$ $\mathrm{nm}$ ) was performed to assess the purity of the porphyrin dyads. Analytical SEC was performed on an HPLC instrument using one $1000 \AA$ column ( $5 \mu \mathrm{L}$, styrene-divinylbenzene copolymer) with THF as eluent $\left(0.8 \mathrm{~mL} / \mathrm{min}\right.$; oven temperature $\left.25^{\circ} \mathrm{C}\right)$. Melting points are uncorrected. Silica gel (40 $\mu \mathrm{m}$ average particle size) was used for column chromatography. Preparative-scale size exclusion chromatography (SEC) was performed using BioRad Bio-beads SX-1 in THF (HPLC grade). THF was freshly distilled from sodium as required. Toluene was distilled from $\mathrm{CaH}_{2}$. $\mathrm{CHCl}_{3}$ was stabilized with $0.8 \%$ ethanol. Anhydrous $\mathrm{MeOH}, \mathrm{CH}_{2} \mathrm{Cl}_{2}$ and $\mathrm{CHCl}_{3}$ (stabilized with $0.8 \% \mathrm{EtOH}$ ) were reagent grade and were used as received. Sonication of crude reaction samples was performed (typically to remove chromatographic residue) with use of a benchtop low-power sonication bath.

Noncommercial Compounds. The following compounds were prepared as described in the literature: dipyrromethanes $\mathbf{1 a},{ }^{18,19} \mathbf{1 c},{ }^{7} \mathbf{1 d}{ }^{18,29}$ and $\mathbf{1 e} ;^{24,31}$ diacyldipyrromethanes $\mathbf{2},{ }^{13} \mathbf{5}^{18,21}$ and $\mathbf{1 7} ;{ }^{18}$ porphyrins $3^{13}$ and $\mathbf{4} ;{ }^{17}$ aldehyde $7{ }^{22,23}$ acid chloride $8 ;{ }^{25}$ and pyridyl thioester $9 .{ }^{26}$

\section{Diacylation Studies: Results.}

Preparation of 1,9-Diacyldipyrromethane 11. Three methods were investigated to prepare the 1,9-diacyldipyrromethane $\mathbf{1 1}$ and the results are summarized in Table S1.

Direct 1,9-Diacylation of Dipyrromethane 1b (All-at-once procedure, Method A). This method is described in the main text.

Sequential 1,9-Diacylation of Dipyrromethane 1b (Method C). This method is described in the main text.

Direct 1,9-Diacylation of Dipyrromethane 1b (Sequential addition procedure, Method B). Since the yield of the desired 1,9-diacyldipyrromethane 11 obtained from the procedure described above (Method A) is low (25\%), we modified this procedure by treating the 
dipyrromethane 1b with EtMgBr and acid chloride $\mathbf{8}$ sequentially and repeatedly. The EtMgBr (4 equiv) was added to the solution of dipyrromethane $1 \mathbf{b}$ in toluene $(\sim 83 \mathrm{mM})$ and the mixture was stirred at room temperature for $15 \mathrm{~min}$, then a solution of acid chloride 8 ( 2 equiv) in toluene was added and the mixture was stirred for $10 \mathrm{~min}$. This procedure was repeated $(\mathrm{EtMgBr}, 1.0$ equiv; acid chloride 8, 0.5 equiv). Under such conditions, the desired 1,9-diacyldipyrromethane 11 was obtained in as high as $41 \%$ yield, while the 1-acyldipyrromethane $\mathbf{1 0}$ was obtained in $30 \%$ yield. This reaction was repeated three times at a $15.0 \mathrm{mmol}$ scale (dipyrromethane $\mathbf{1 b}$ ) and the product 11 was obtained in an average yield of $34 \%$ (totally $\sim 12 \mathrm{~g}$ ). The experimental procedure for the direct diacylation of $\mathbf{1 b}$ is given as follows:

\section{1,9-Bis(3,5-di-tert-butylbenzoyl)-5-[4-(5,5-dimethyl-1,3-dioxan-2-}

yl)]phenyldipyrromethane (11). A solution of $\mathbf{1 b}(5.05 \mathrm{~g}, 15.0 \mathrm{mmol})$ in toluene $(180 \mathrm{~mL}) \mathrm{was}$ treated with EtMgBr $(60.0 \mathrm{~mL}, 60 \mathrm{mmol}, 1.0 \mathrm{M}$ solution in THF) at room temperature for 15 min. A solution of $8(7.58 \mathrm{~g}, 30.0 \mathrm{mmol})$ in toluene $(20 \mathrm{~mL})$ was added dropwise and the reaction mixture was stirred at room temperature for $10 \mathrm{~min}$. Additional EtMgBr $(15.0 \mathrm{~mL}, 15.0$ mmol, 1.0 M solution in THF) and a solution of $8(1.90 \mathrm{~g}, 7.50 \mathrm{mmol})$ in toluene $(5.0 \mathrm{~mL})$ were added likewise. After stirring at room temperature for $10 \mathrm{~min}$, the reaction mixture was quenched with aqueous $\mathrm{NH}_{4} \mathrm{Cl}$. The usual workup and chromatography [silica, hexanes/ethyl acetate (4:1)] gave most of the $\mathbf{1 1}$ in the first fraction, which was chromatographed again [silica, $\mathrm{CH}_{2} \mathrm{Cl}_{2}$ /ethyl acetate (90:10)], affording 11 as a brown solid (4.71 g, 41\%). The characterization data were identical with those obtained from method A. The second fraction was concentrated to afford compound $10(2.5 \mathrm{~g}, 30 \%)$. The characterization data were consistent with those obtained from Method A. Note that the elution order of $\mathbf{1 1}$ and $\mathbf{1 0}$ is reversed from the typical case upon acylation of dipyrromethanes. This reaction was repeated three times at the same scale and the average yield of the diacyldipyrromethane 11 was $34 \%$ (totally $\sim 12 \mathrm{~g}$ ), while the average yield of the 1 -acyldipyrromethane $\mathbf{1 0}$ was $32 \%$ (totally $7.96 \mathrm{~g}$ ).

Table S1: Conditions for diacylation of dipyrromethane $\mathbf{1 b .}$

\begin{tabular}{|c|c|c|c|c|}
\hline \multirow[t]{2}{*}{ Method } & \multirow[t]{2}{*}{ Acylation procedure } & \multirow{2}{*}{$\begin{array}{l}\text { Chromatography } \\
\text { processes }\end{array}$} & \multicolumn{2}{|c|}{$\begin{array}{l}\text { Yield of acyl- } \\
\text { dipyrromethanes }\end{array}$} \\
\hline & & & $\begin{array}{l}1 \text {-acyl } \\
(\mathbf{1 0})\end{array}$ & $\begin{array}{l}\text { 1,9-diacyl } \\
\text { (11) }\end{array}$ \\
\hline $\mathrm{A}$ & $\begin{array}{l}\text { Direct 1,9-diacylation:(All-at-once } \\
\text { procedure; in text) }\end{array}$ & One column & $47 \%$ & $25 \%$ \\
\hline $\mathrm{B}$ & $\begin{array}{l}\text { Direct 1,9-diacylation: (Sequential } \\
\text { addition procedure) }^{b}\end{array}$ & Two columns & $\begin{array}{c}30 \% \\
(32 \%)^{c}\end{array}$ & $\begin{array}{c}41 \% \\
(34 \%)^{c}\end{array}$ \\
\hline $\mathrm{C}$ & $\begin{array}{l}\text { Sequential acylation (in text): } \\
\text { (1) Monoacylation } \\
\text { (2) Second acylation }\end{array}$ & $\begin{array}{l}\text { One column } \\
\text { One column }\end{array}$ & $\begin{array}{c}60 \% \\
--- \\
\end{array}$ & $58 \%$ \\
\hline
\end{tabular}

${ }^{a}$ The dipyrromethane $\mathbf{1 b}$ was treated with $\mathrm{EtMgBr}$ ( 5 equiv, $30 \mathrm{~min}$ ) followed by acid chloride $\mathbf{8}$ ( 2.5 equiv, $10 \mathrm{~min}$ ) all-at once. ${ }^{b}$ The dipyrromethane $\mathbf{1 b}$ was sequentially treated with $\mathrm{EtMgBr}$ followed by acid chloride 8 at the ratio of 4.0 equiv, 2.0 equiv and 1.0 equiv, 0.5 equiv respectively. ${ }^{c}$ Average yields upon repeating the reaction three times at the same scale (15.0 mmol of 1b). ${ }^{d} 9$-Acylation of the 1-acyldipyrromethane 10. The overall yield of 1,9diacyldipyrromethane $\mathbf{1 1}$ was $35 \%$ from dipyrromethane $\mathbf{1 b}$. 


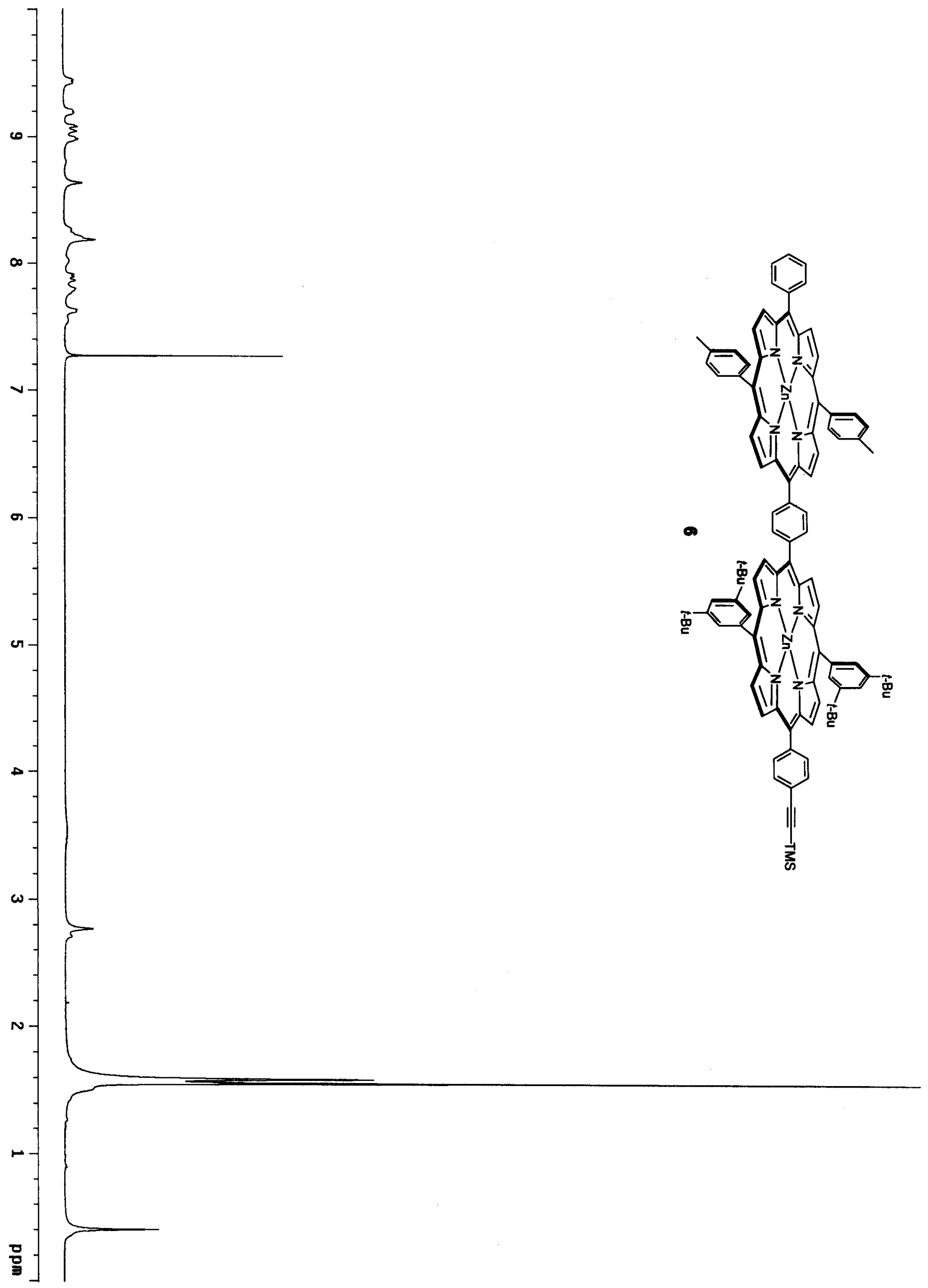




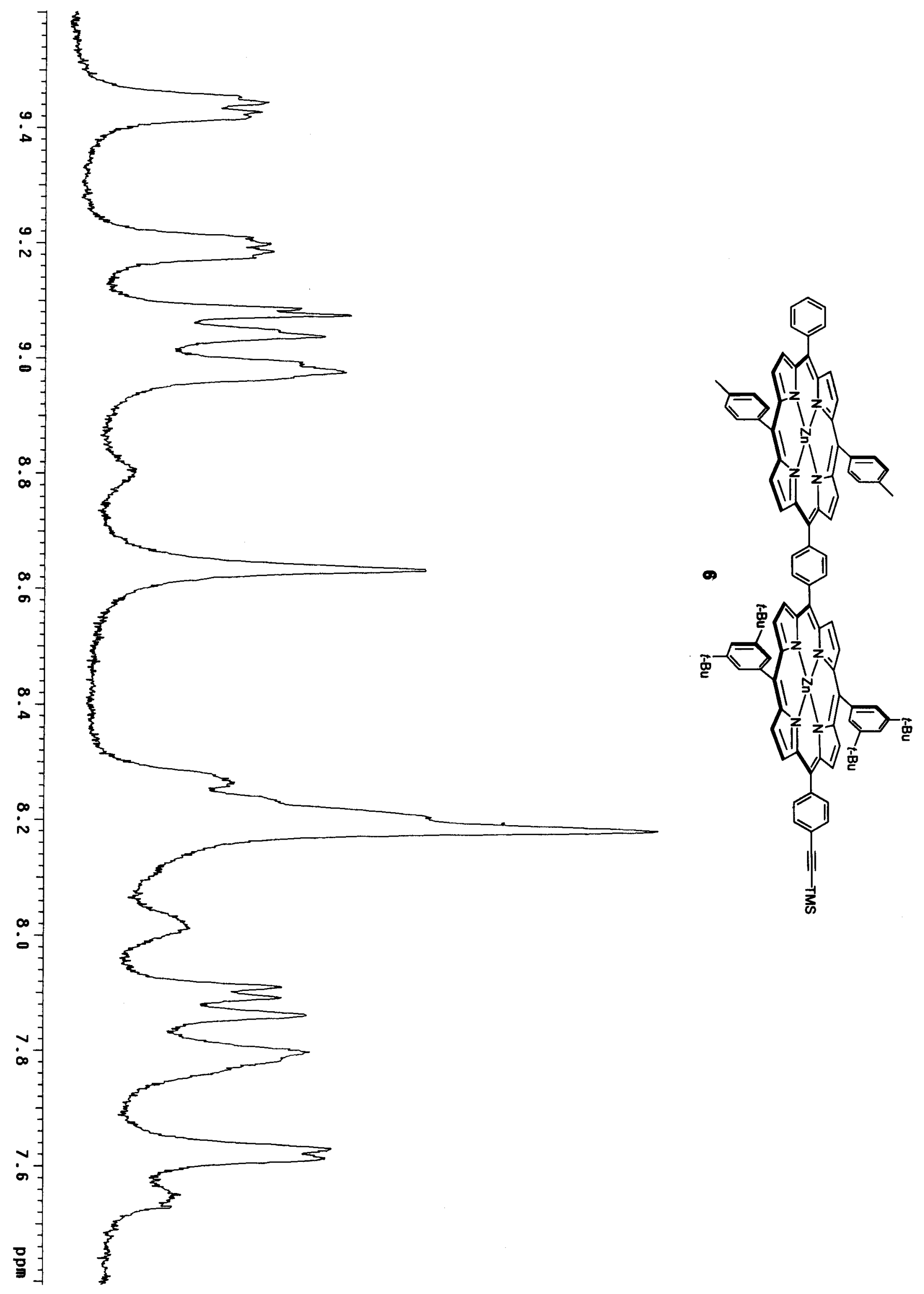




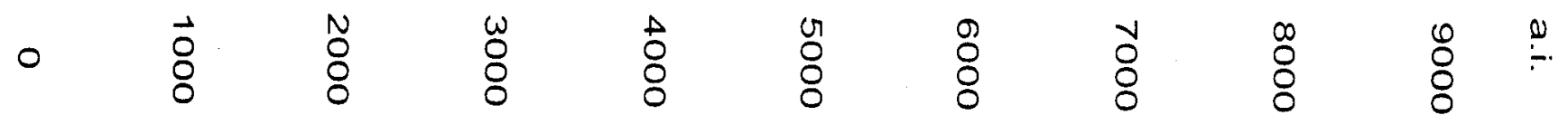

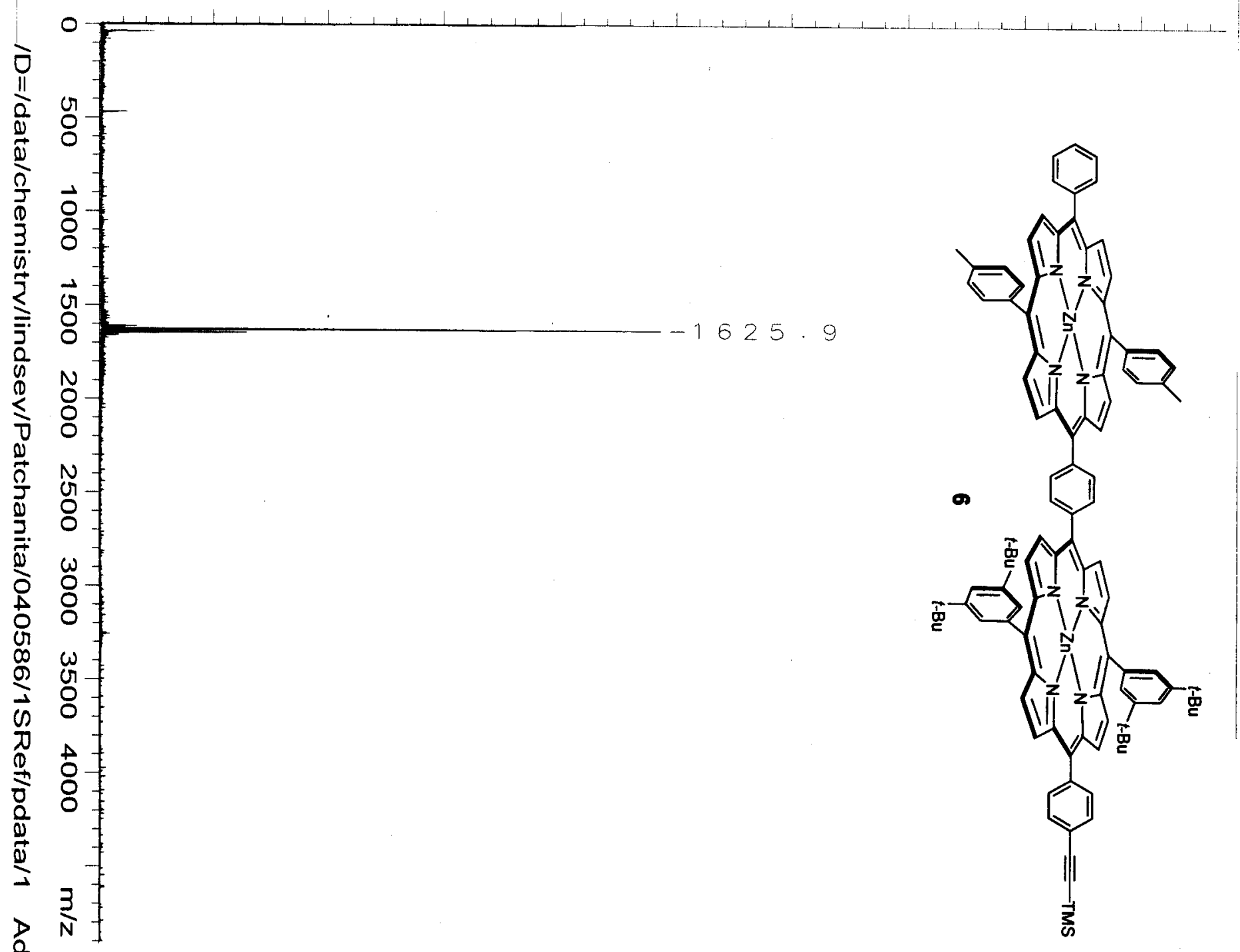

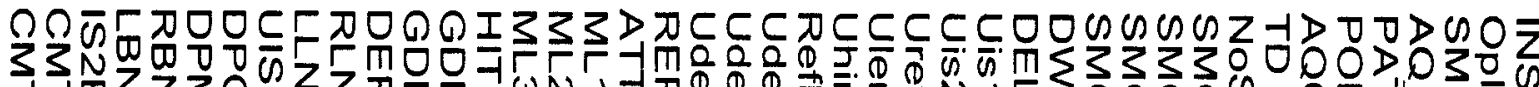

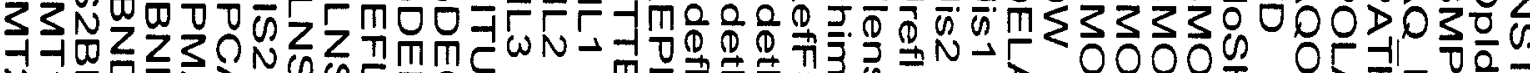

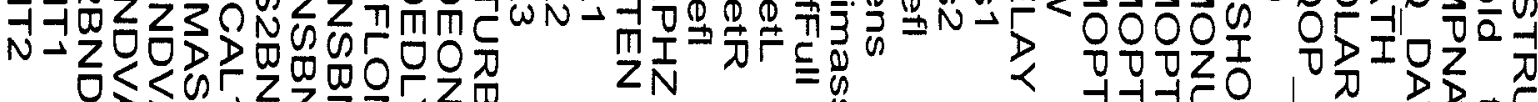

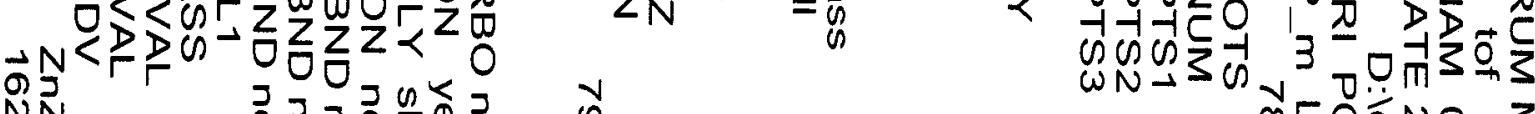

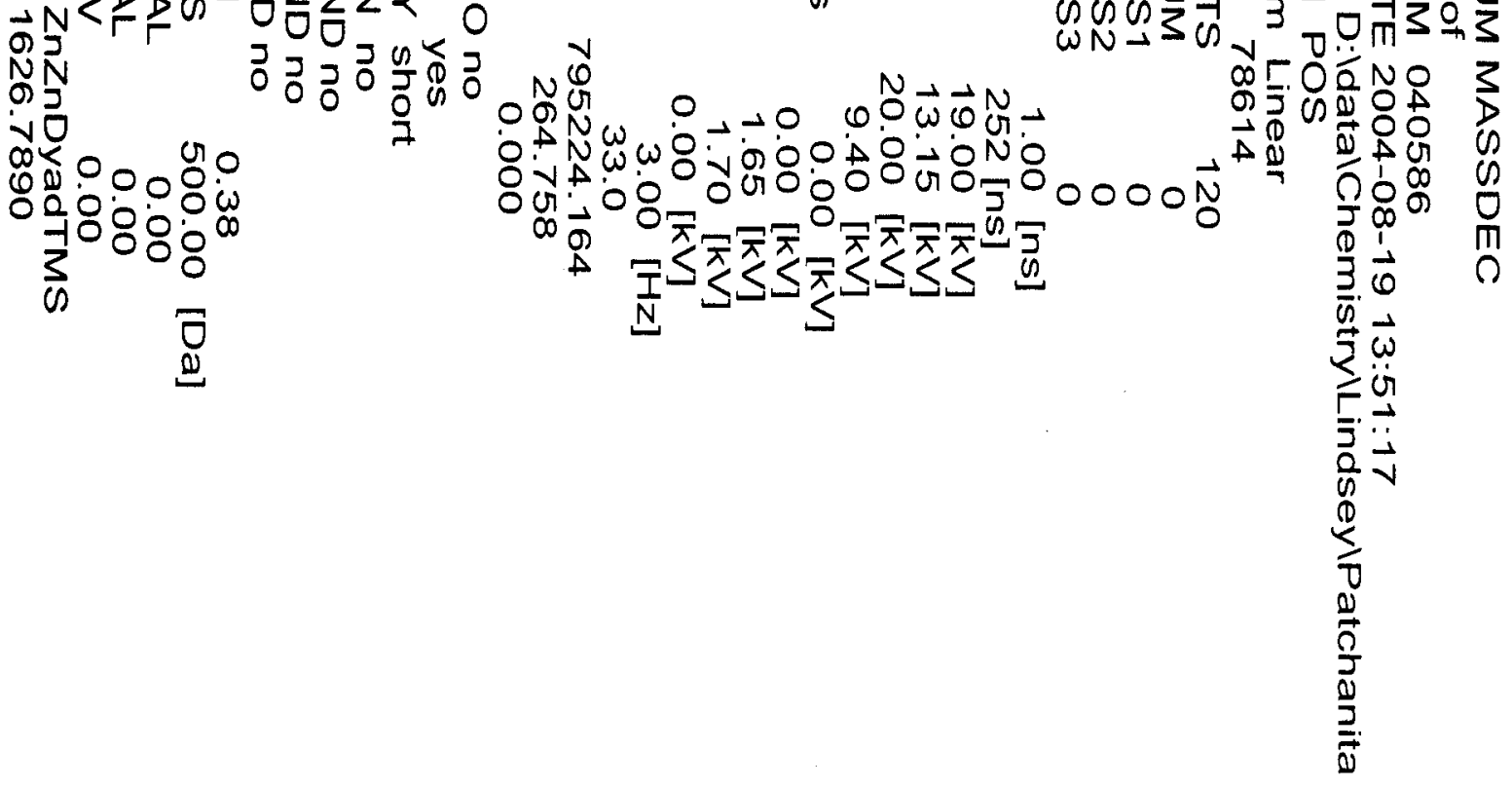




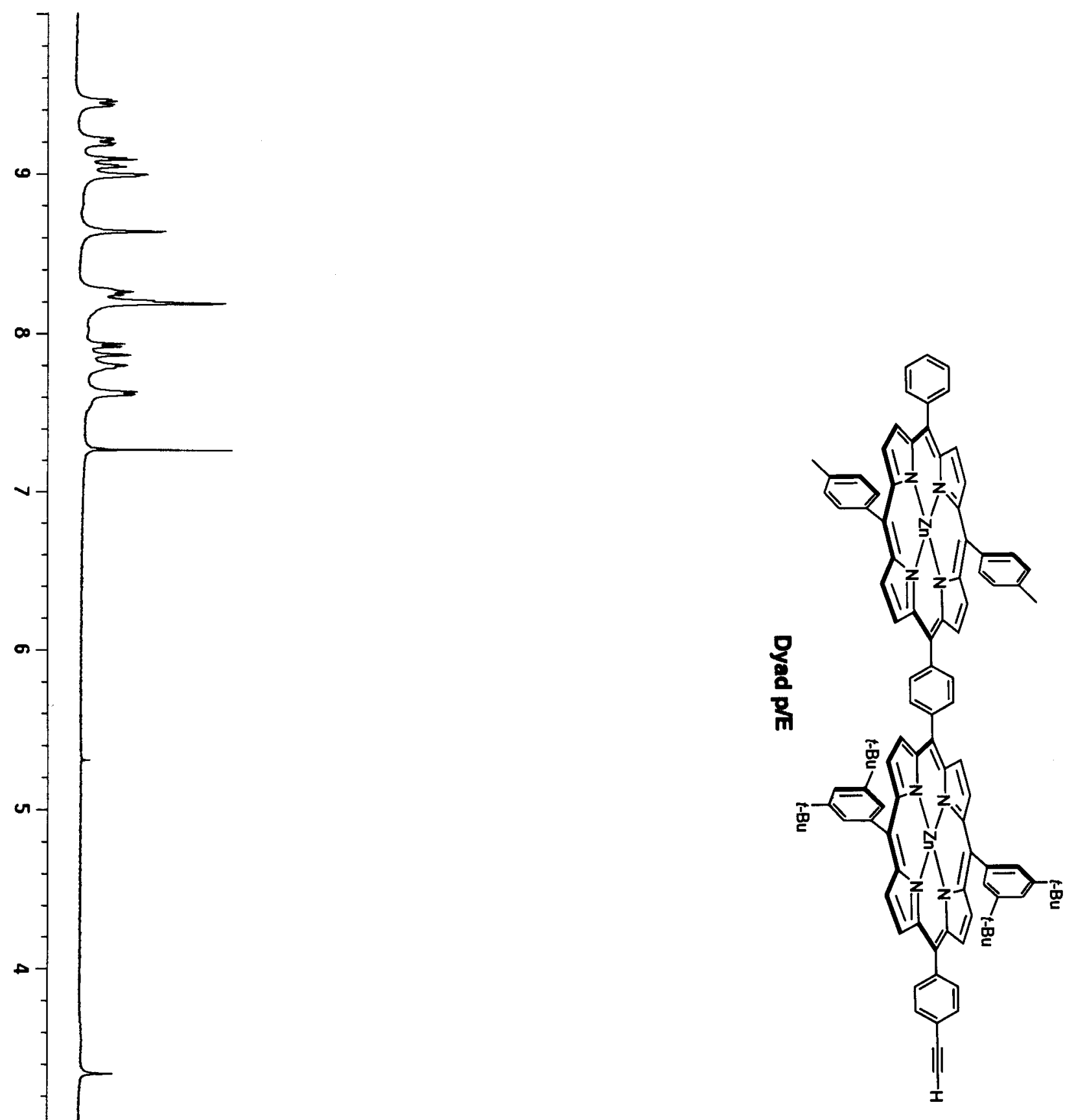




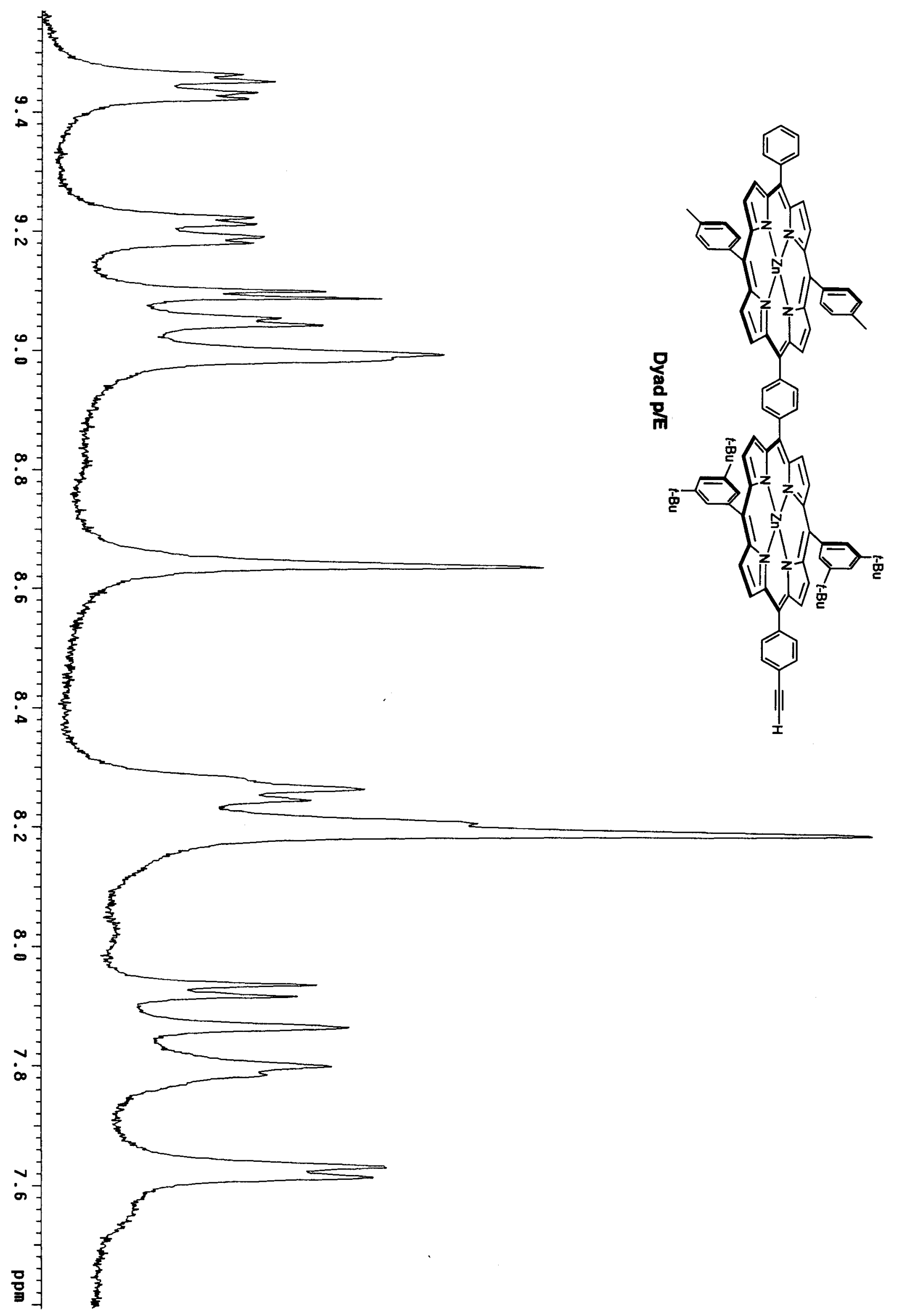




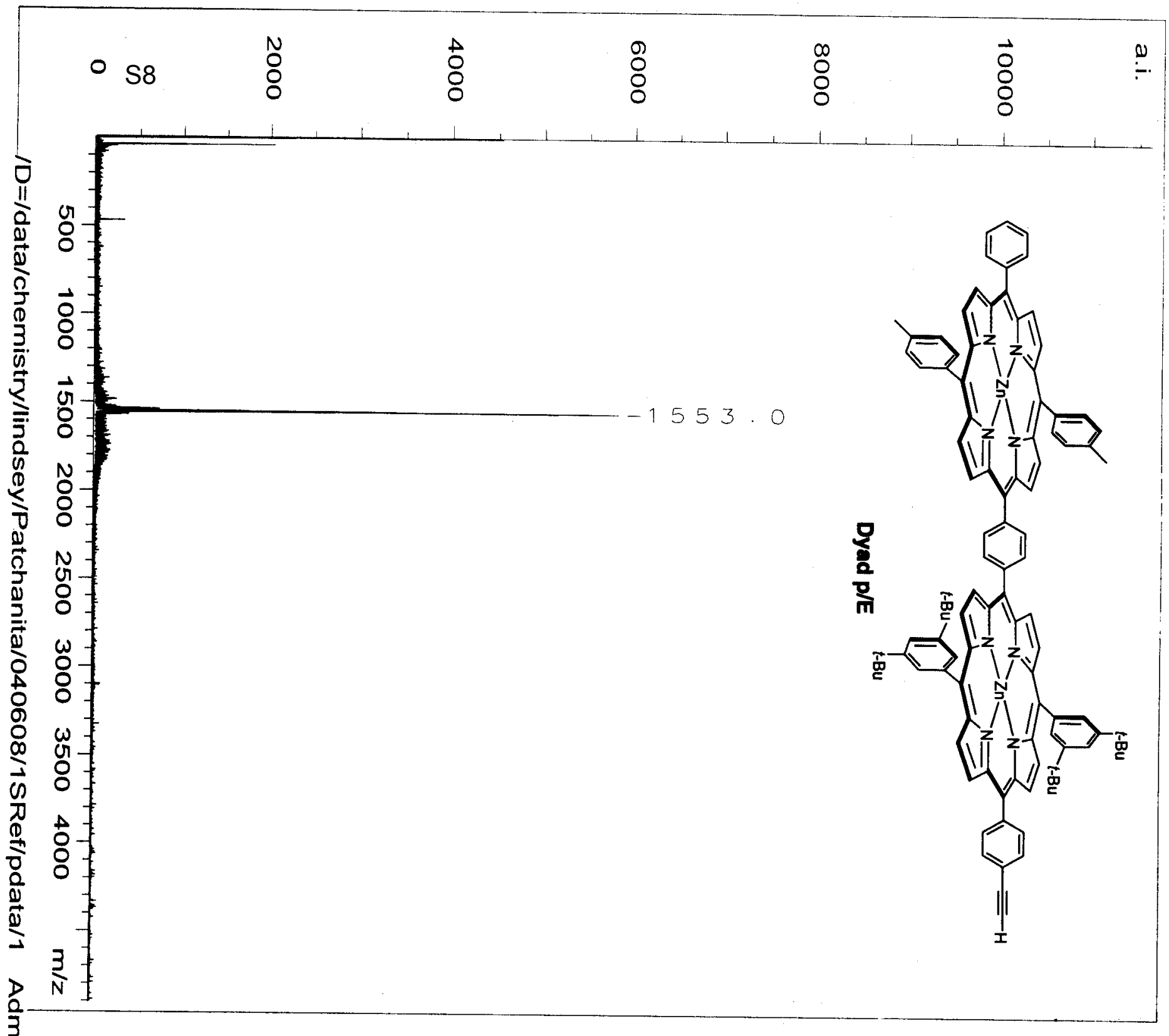

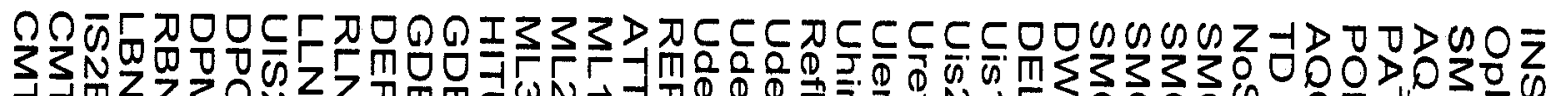

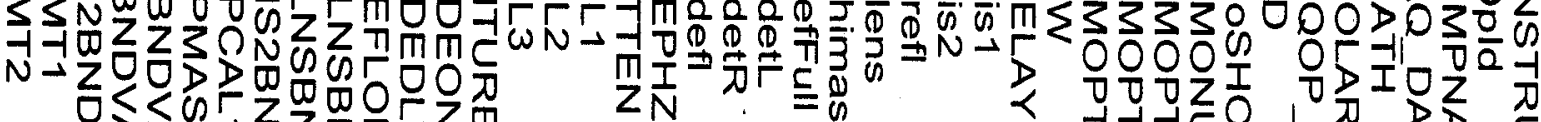

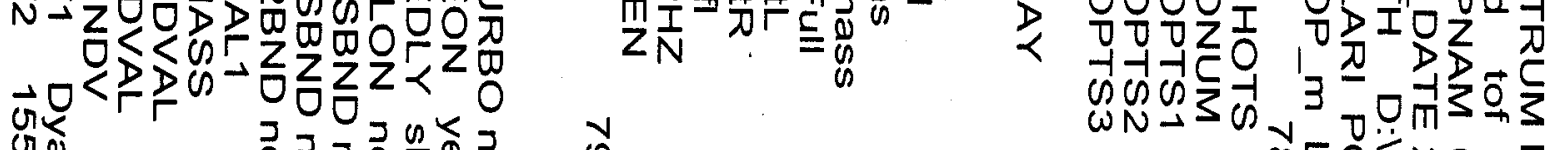

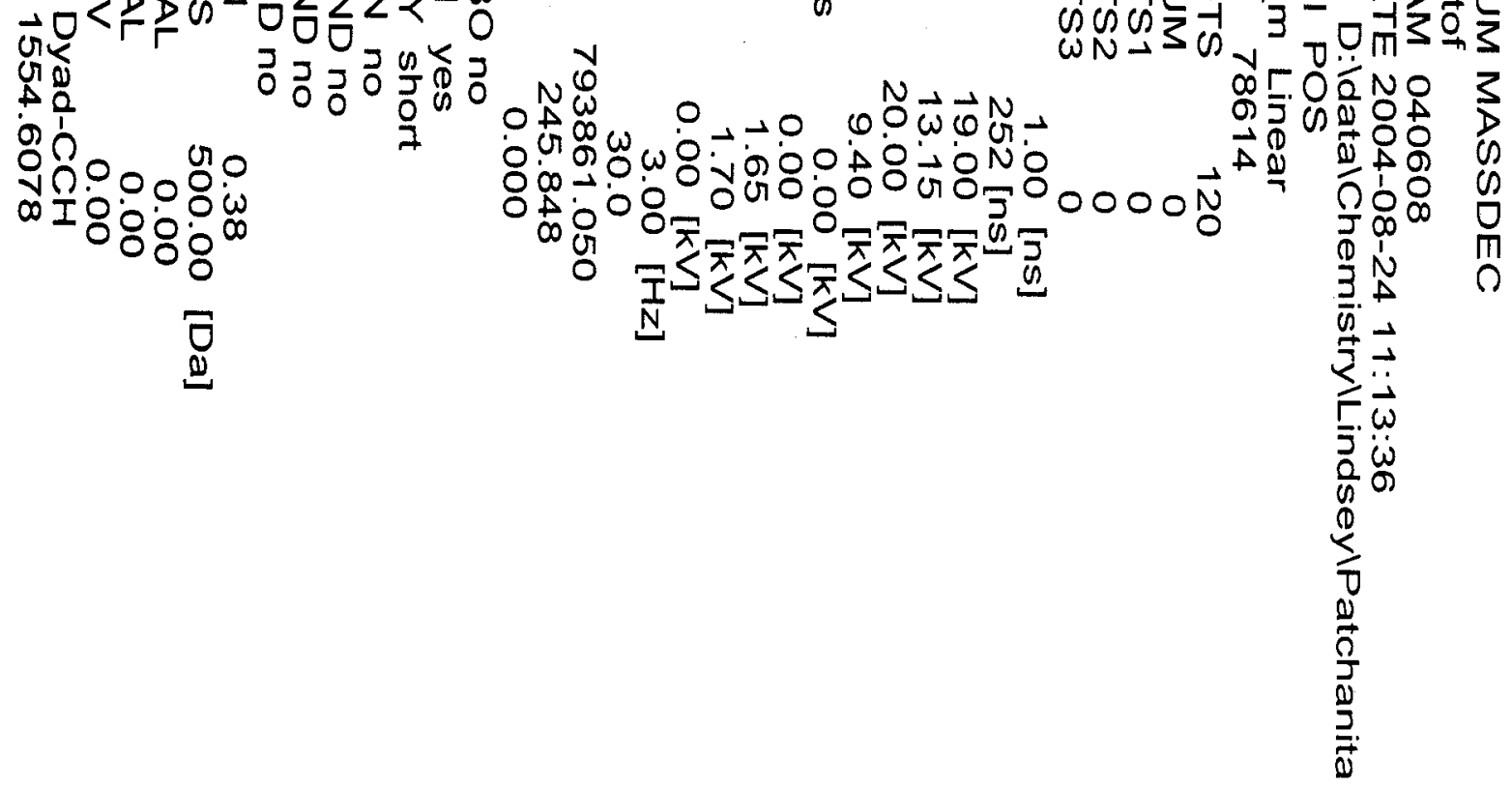




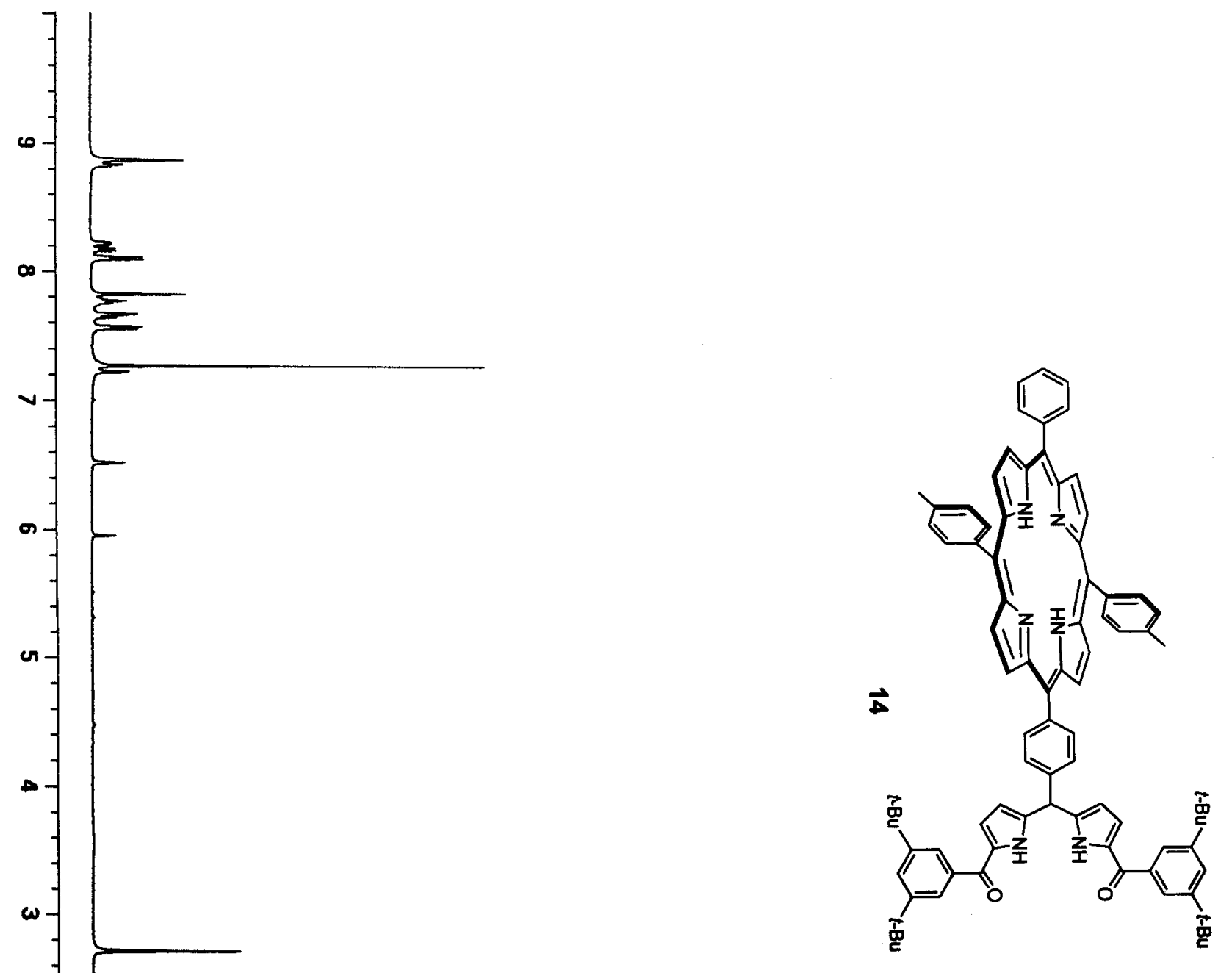



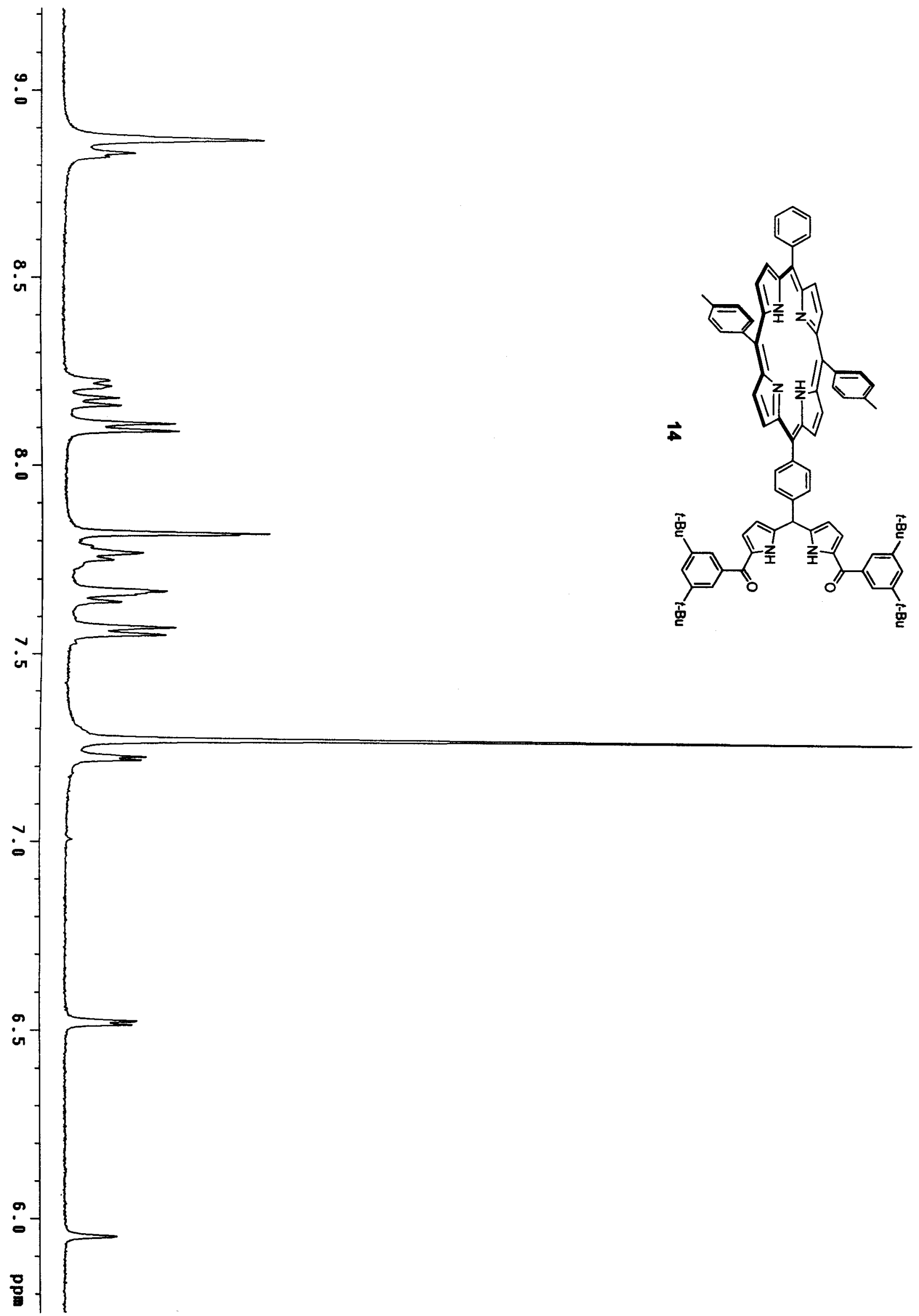


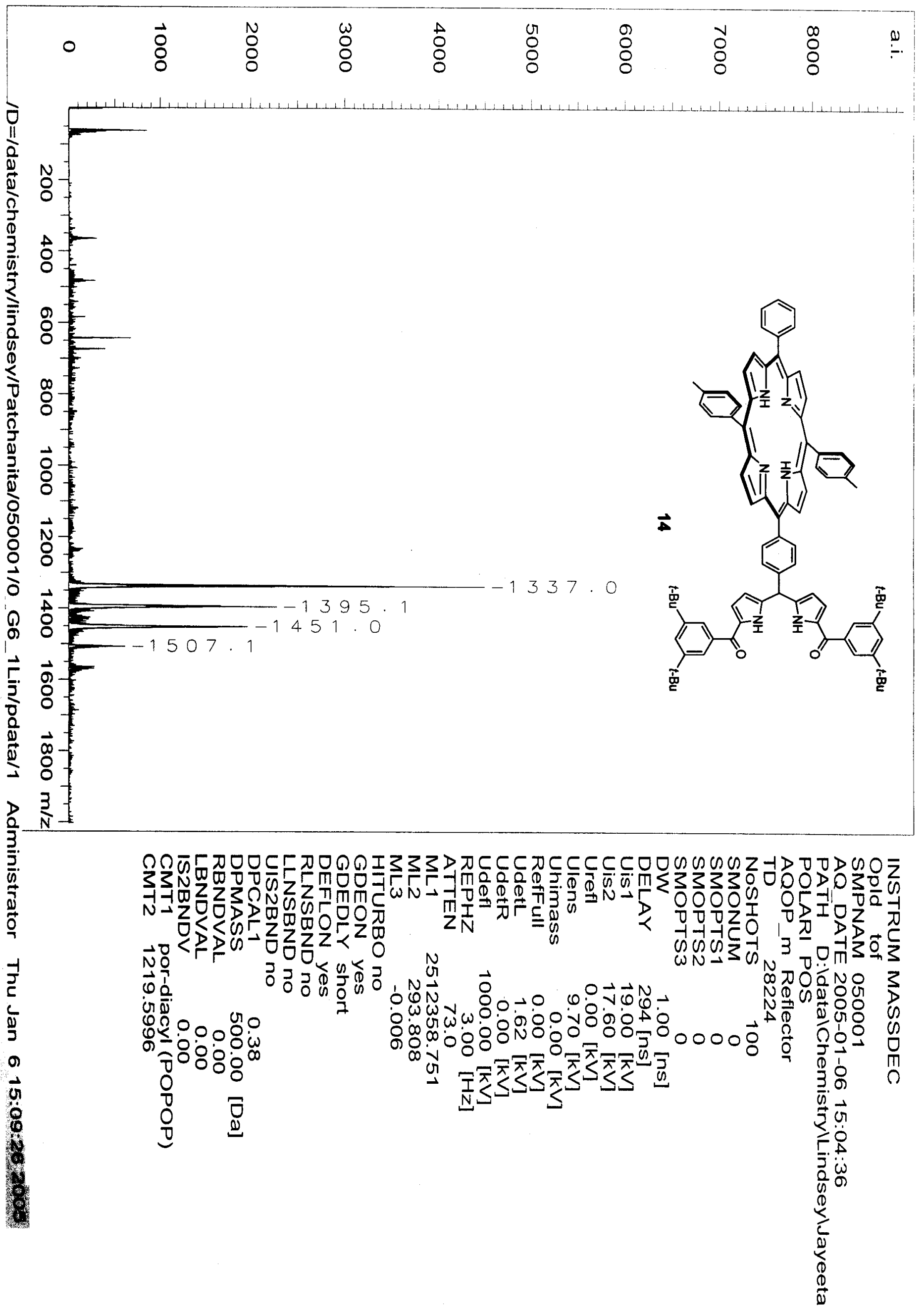




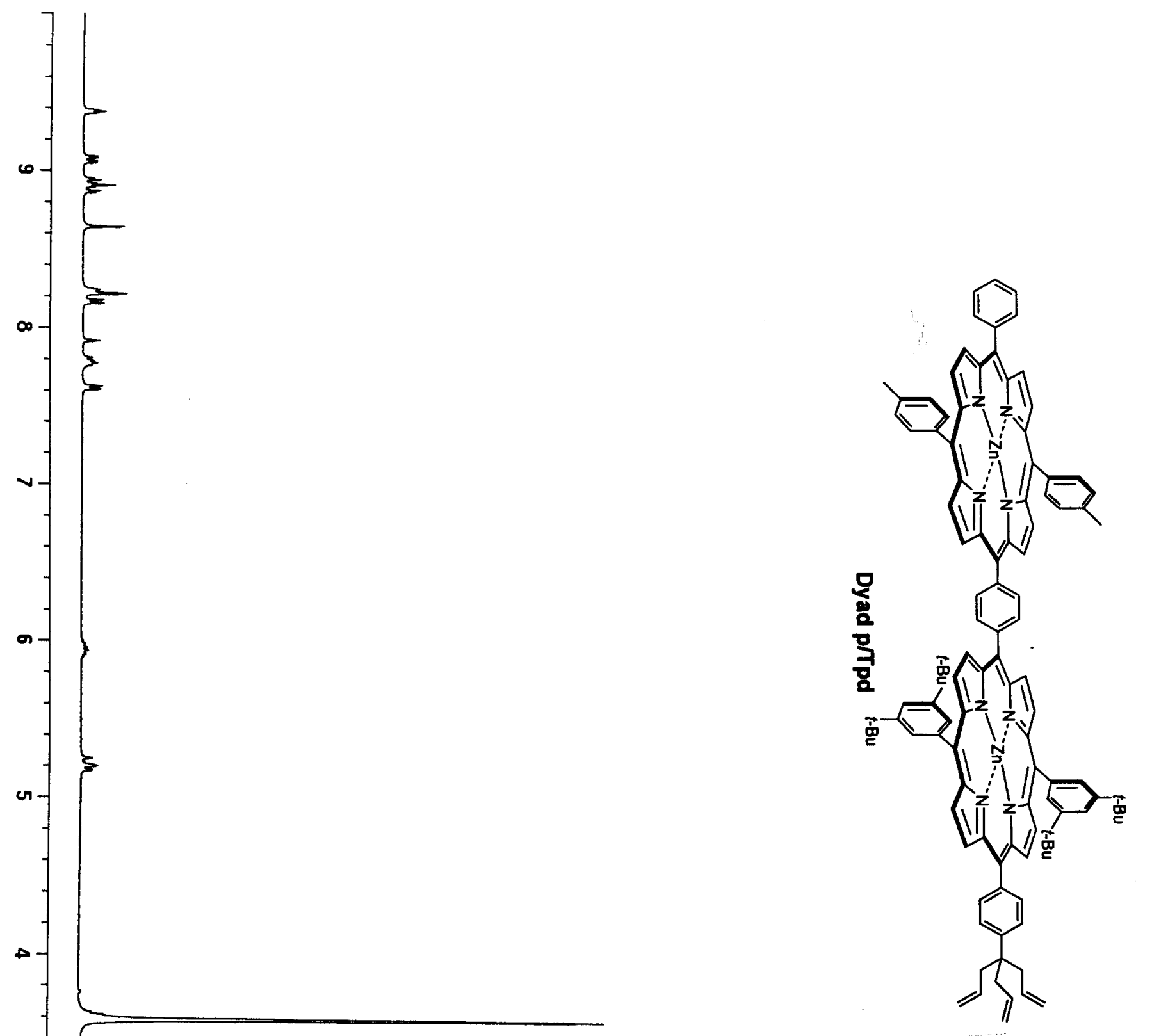

ㅁ. 


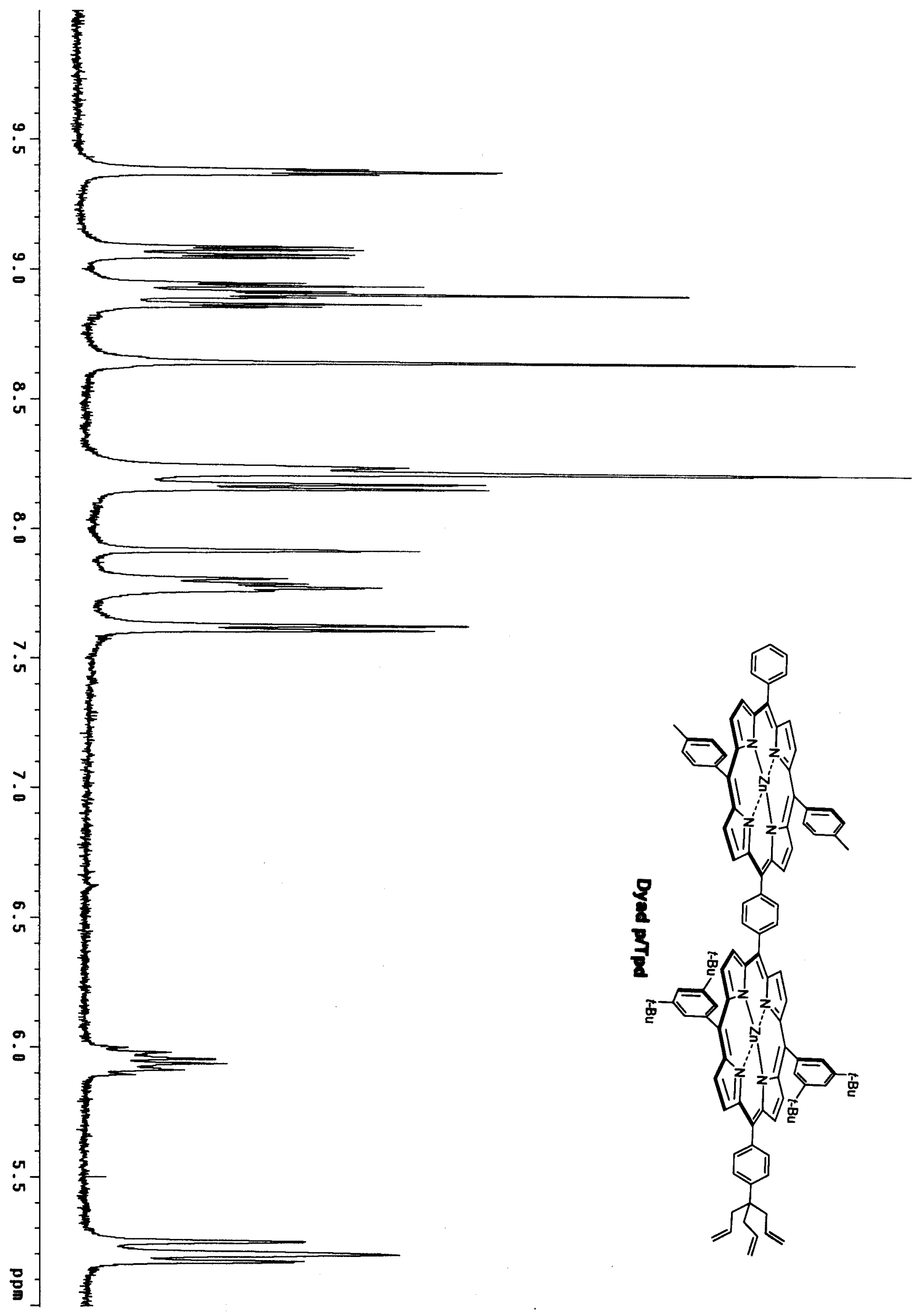




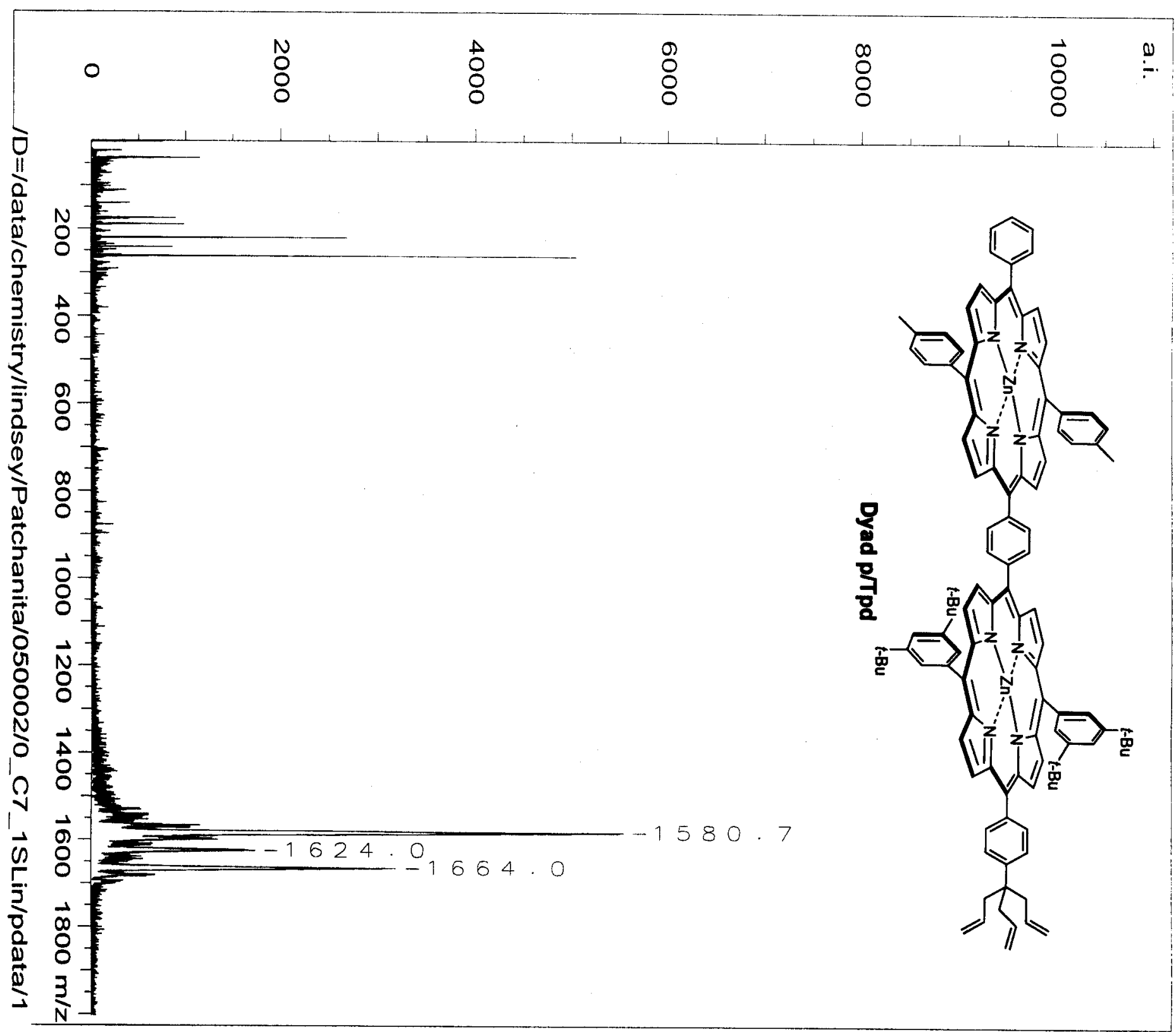

2
$\frac{2}{3}$
$\frac{3}{0}$
$\frac{0}{0}$
$\frac{0}{0}$
$\frac{0}{7}$

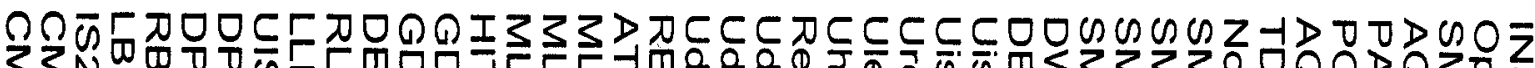

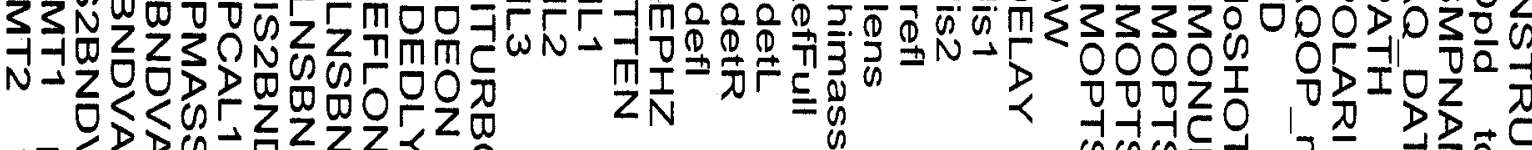

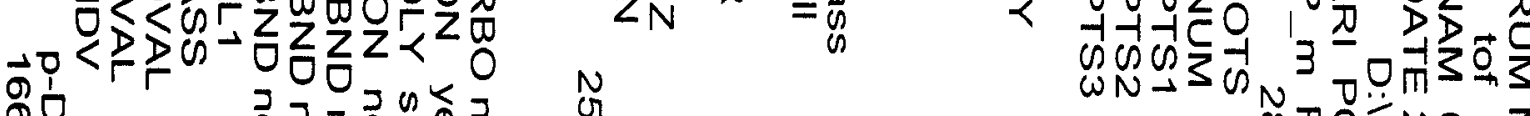

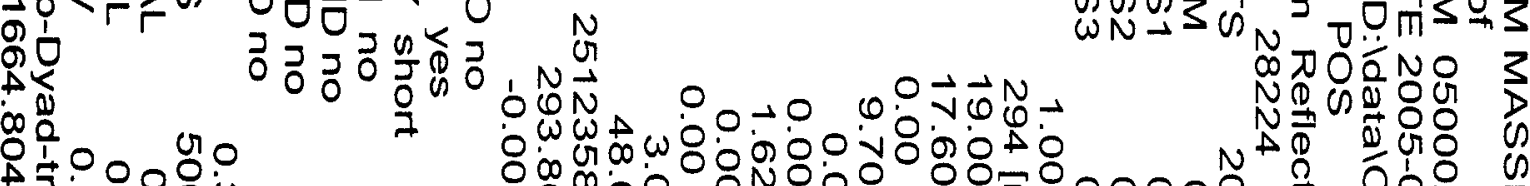

כִ

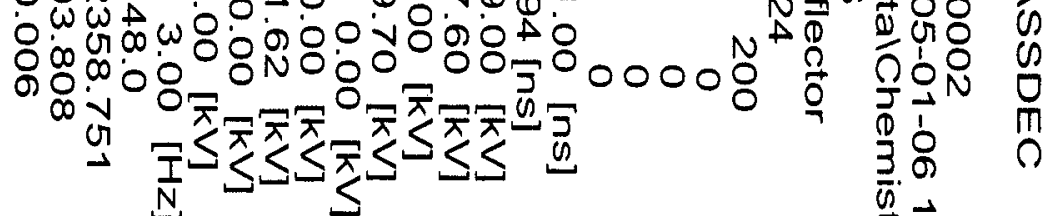

믐

 


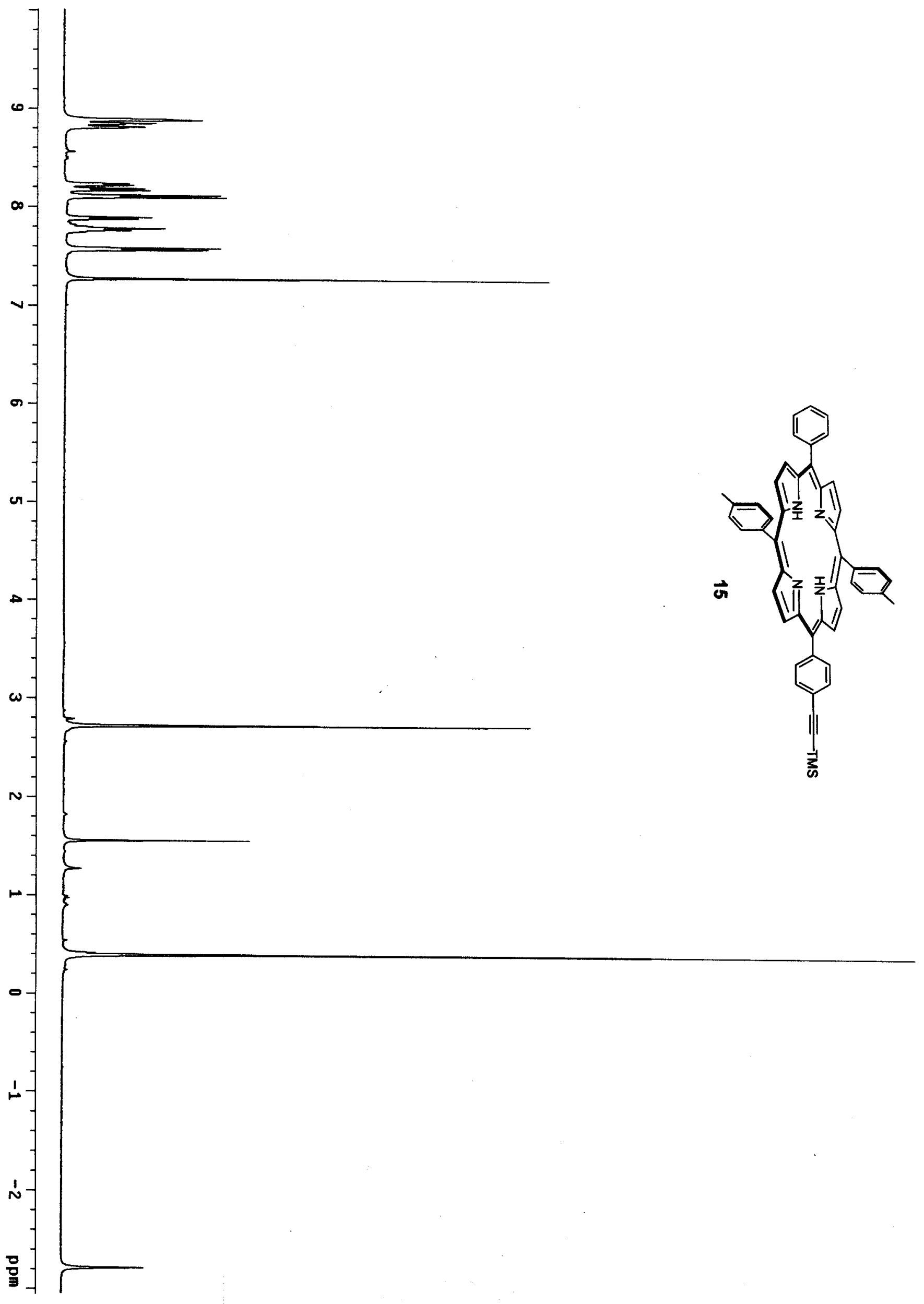




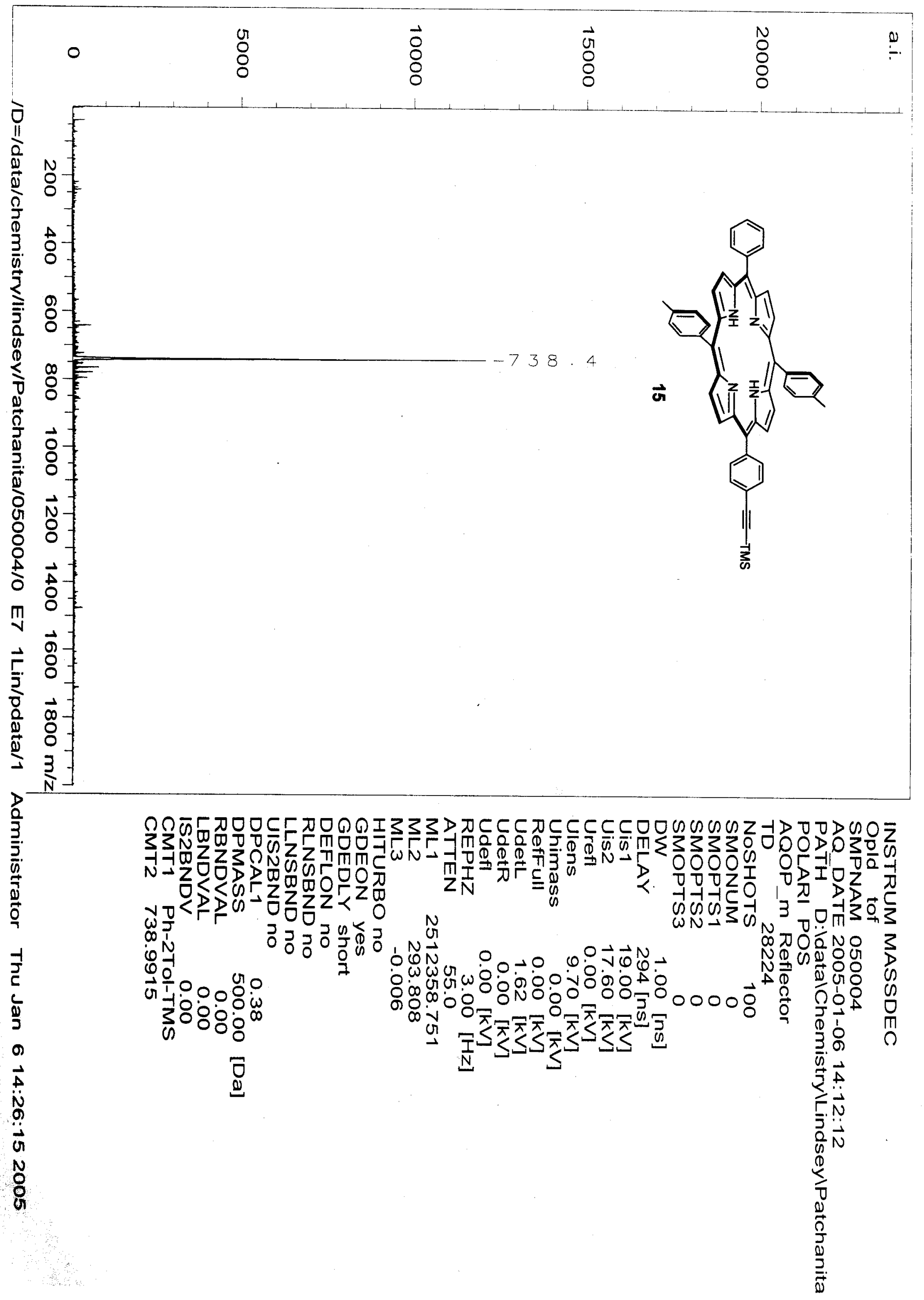




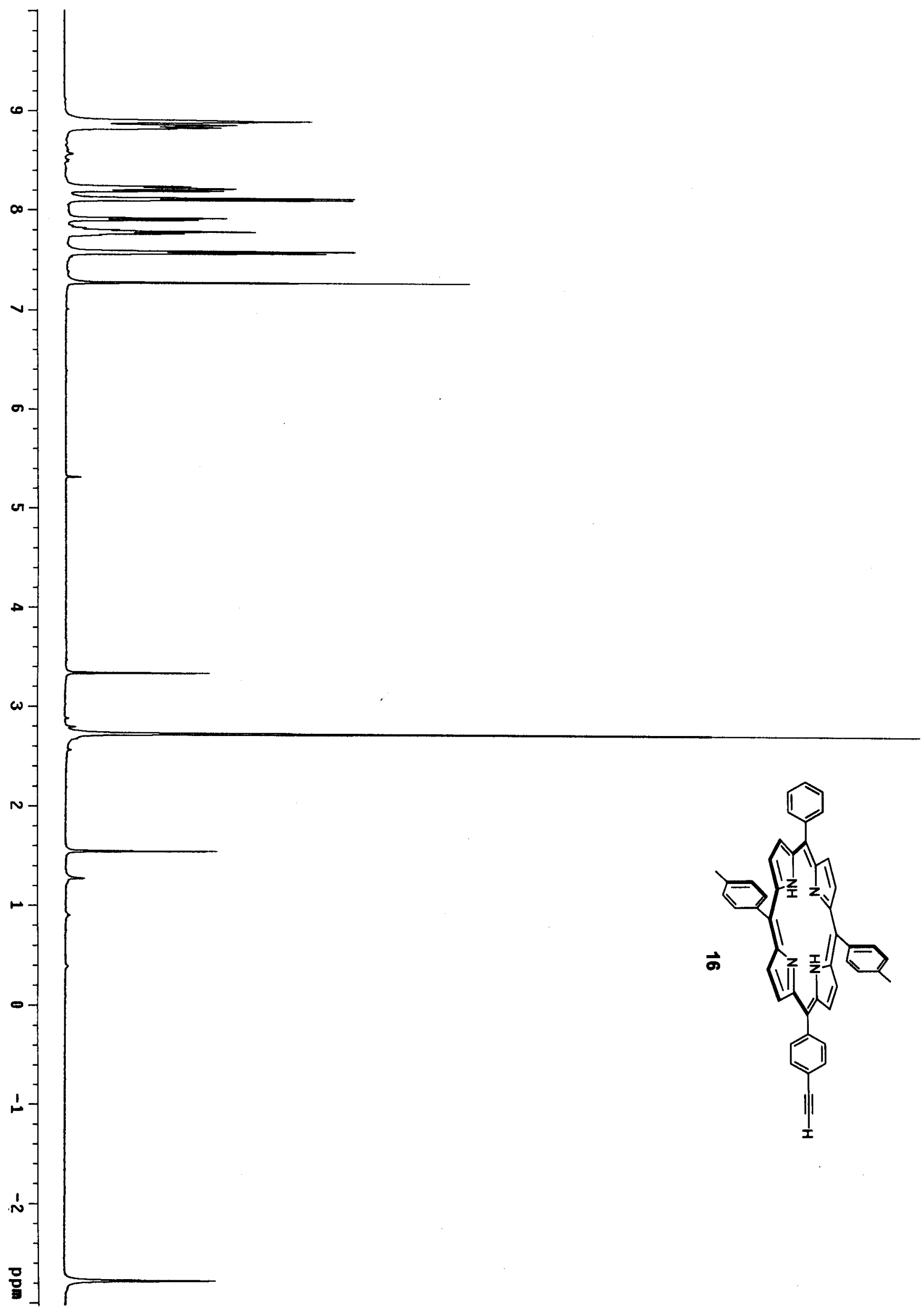




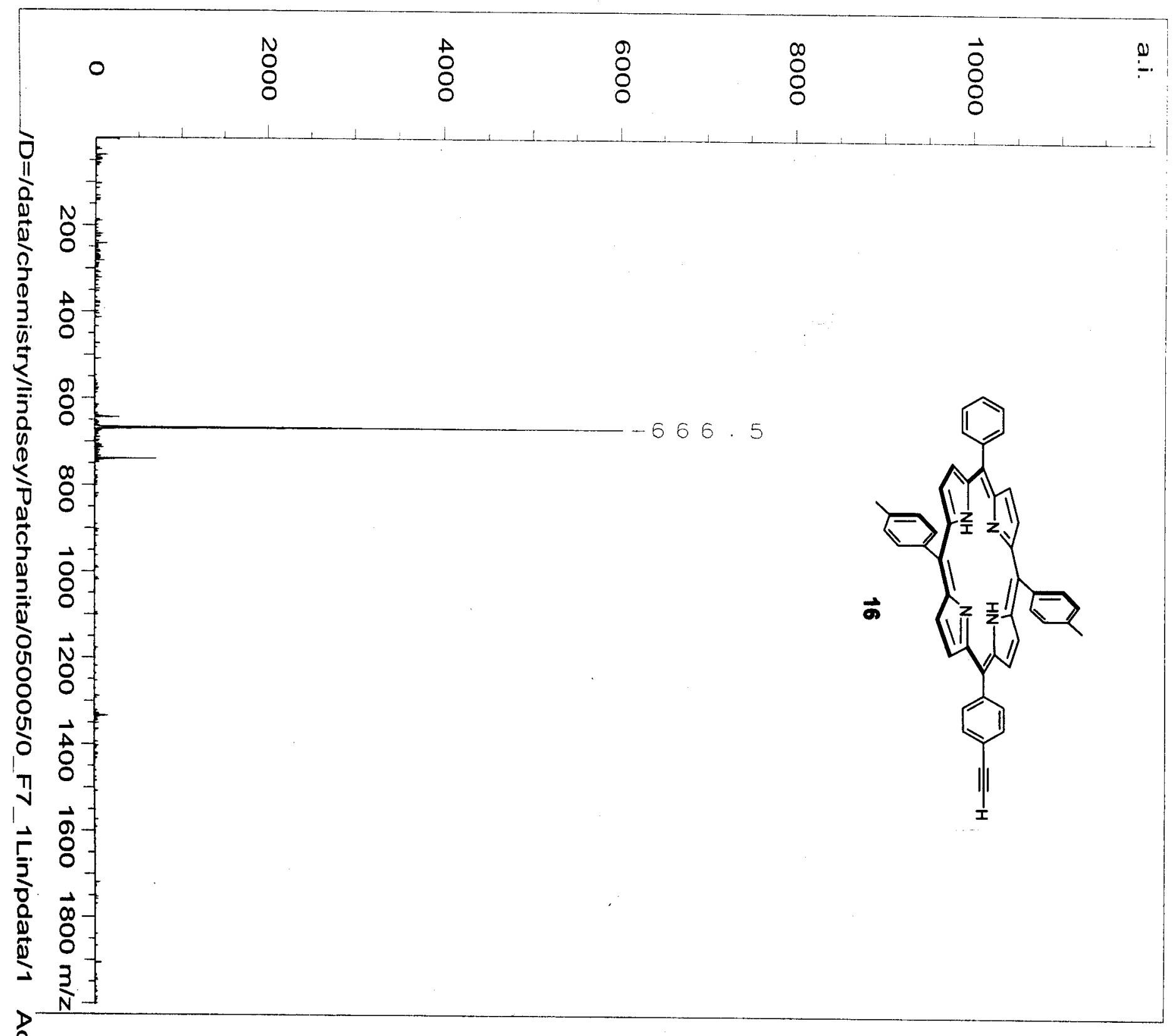

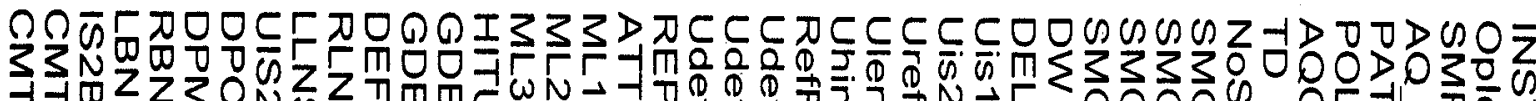

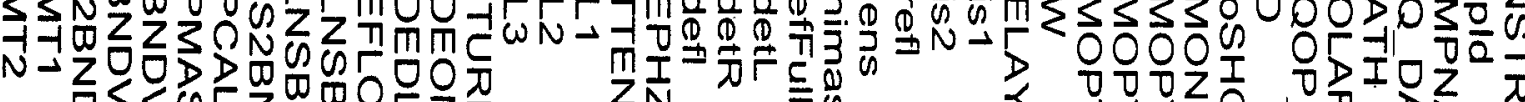

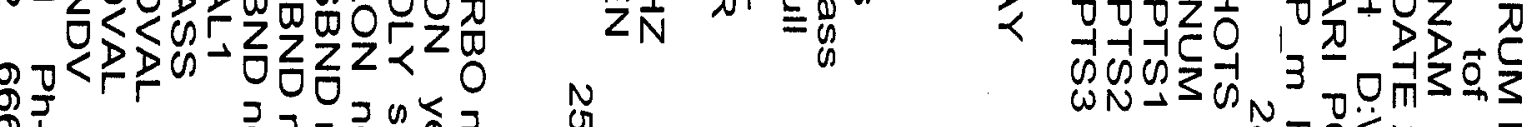

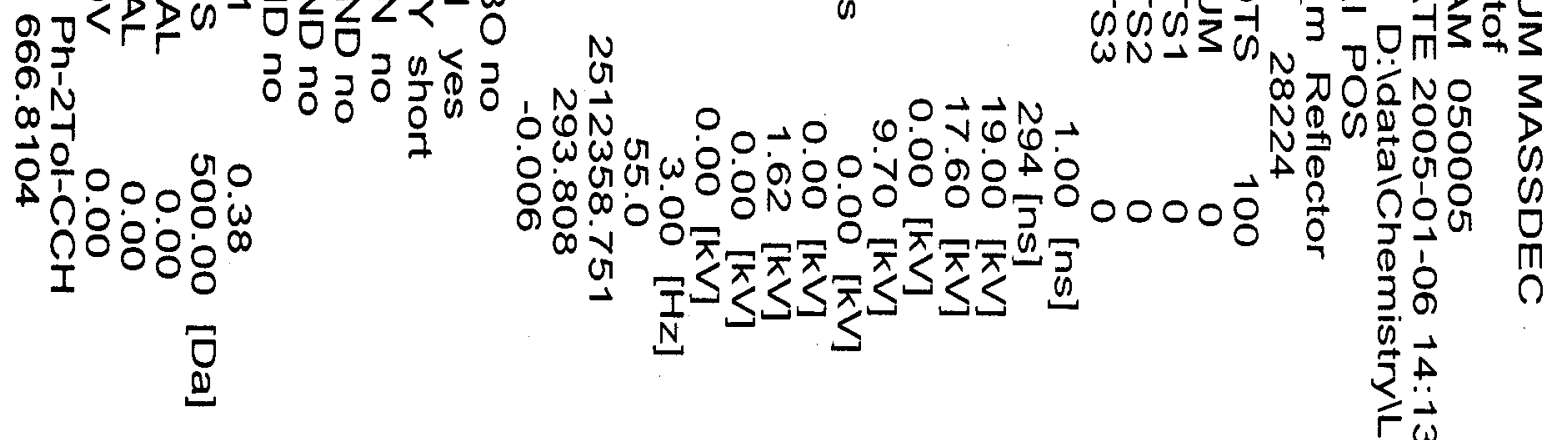

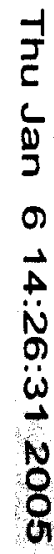

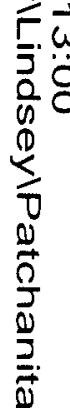




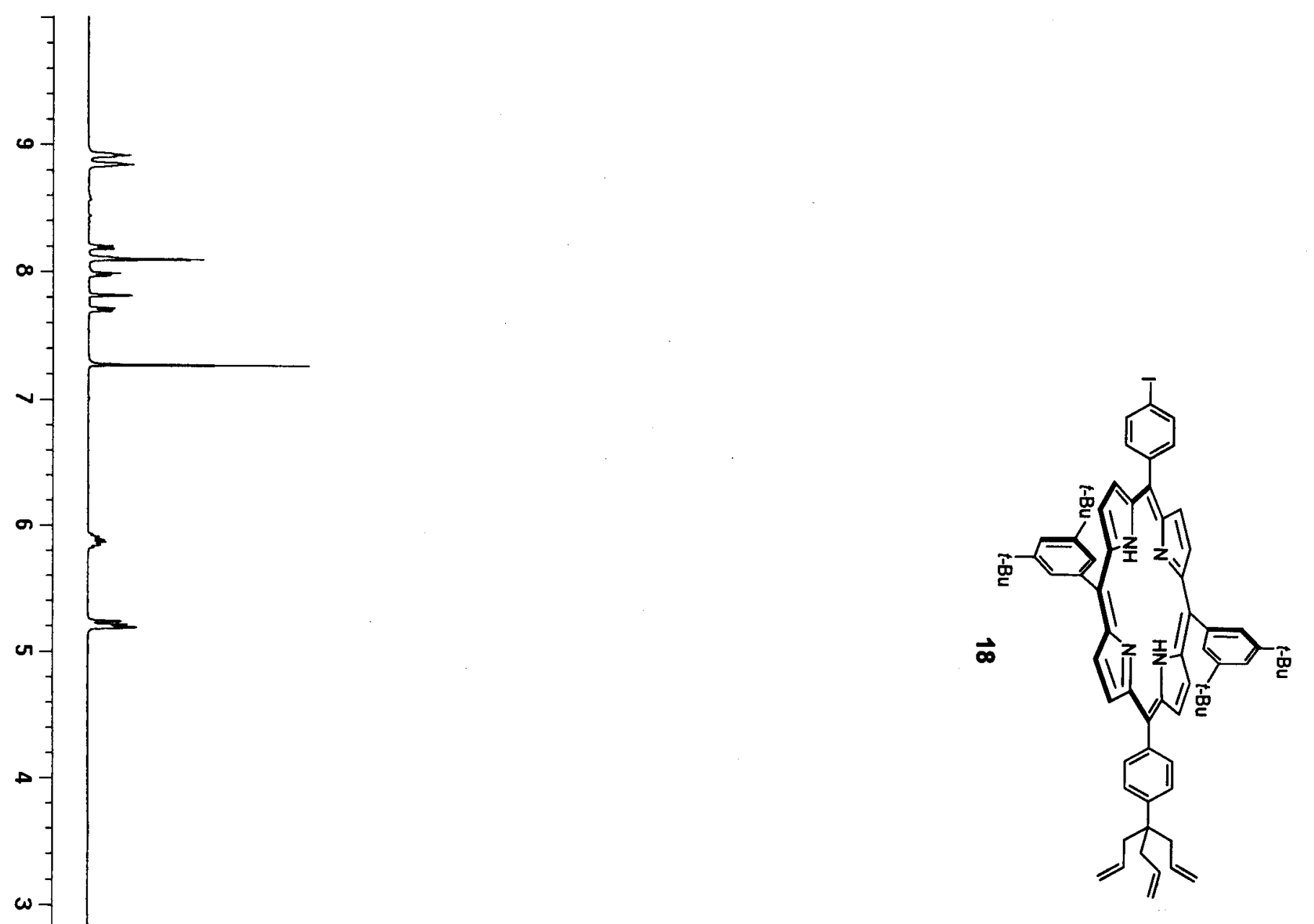




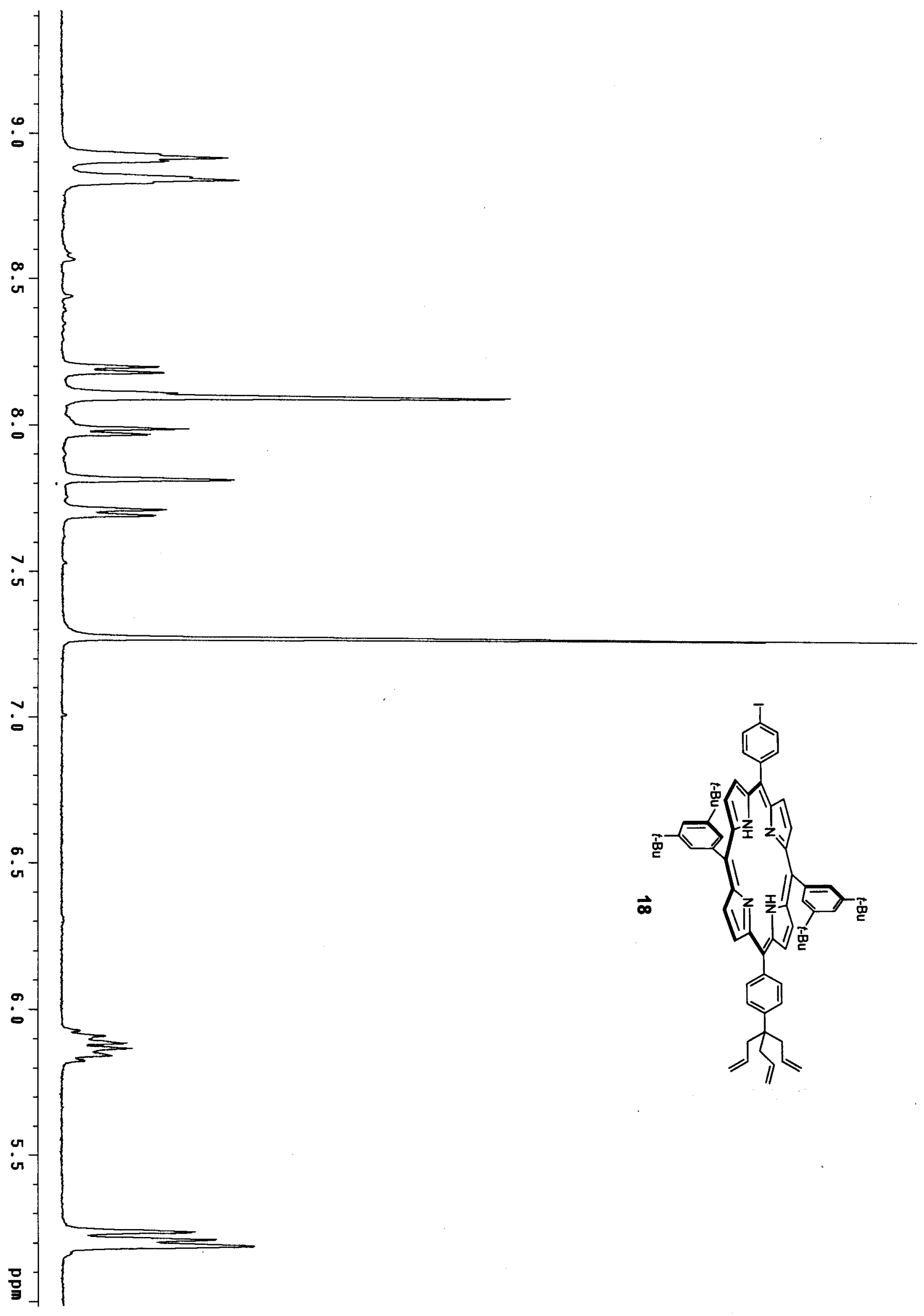




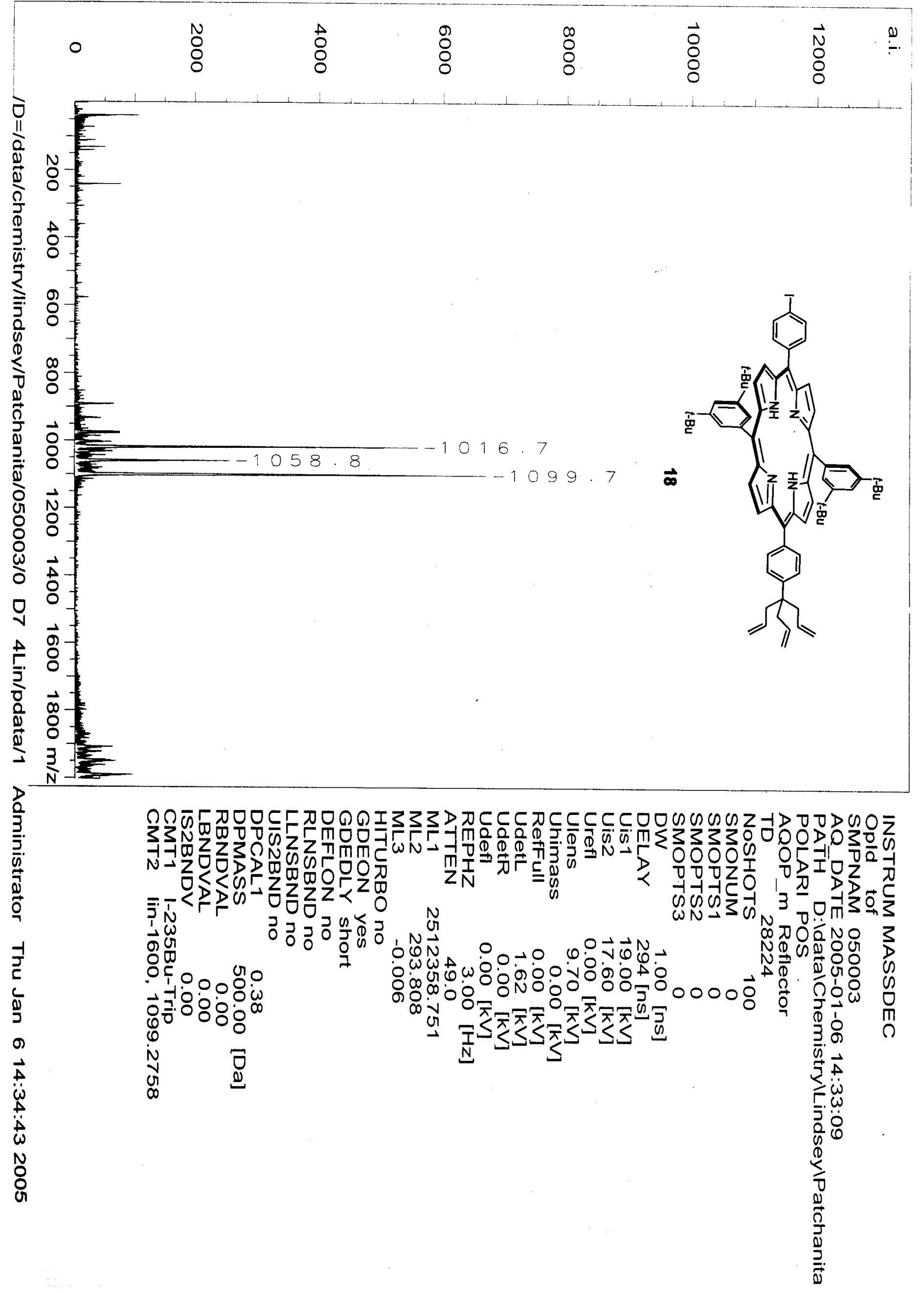




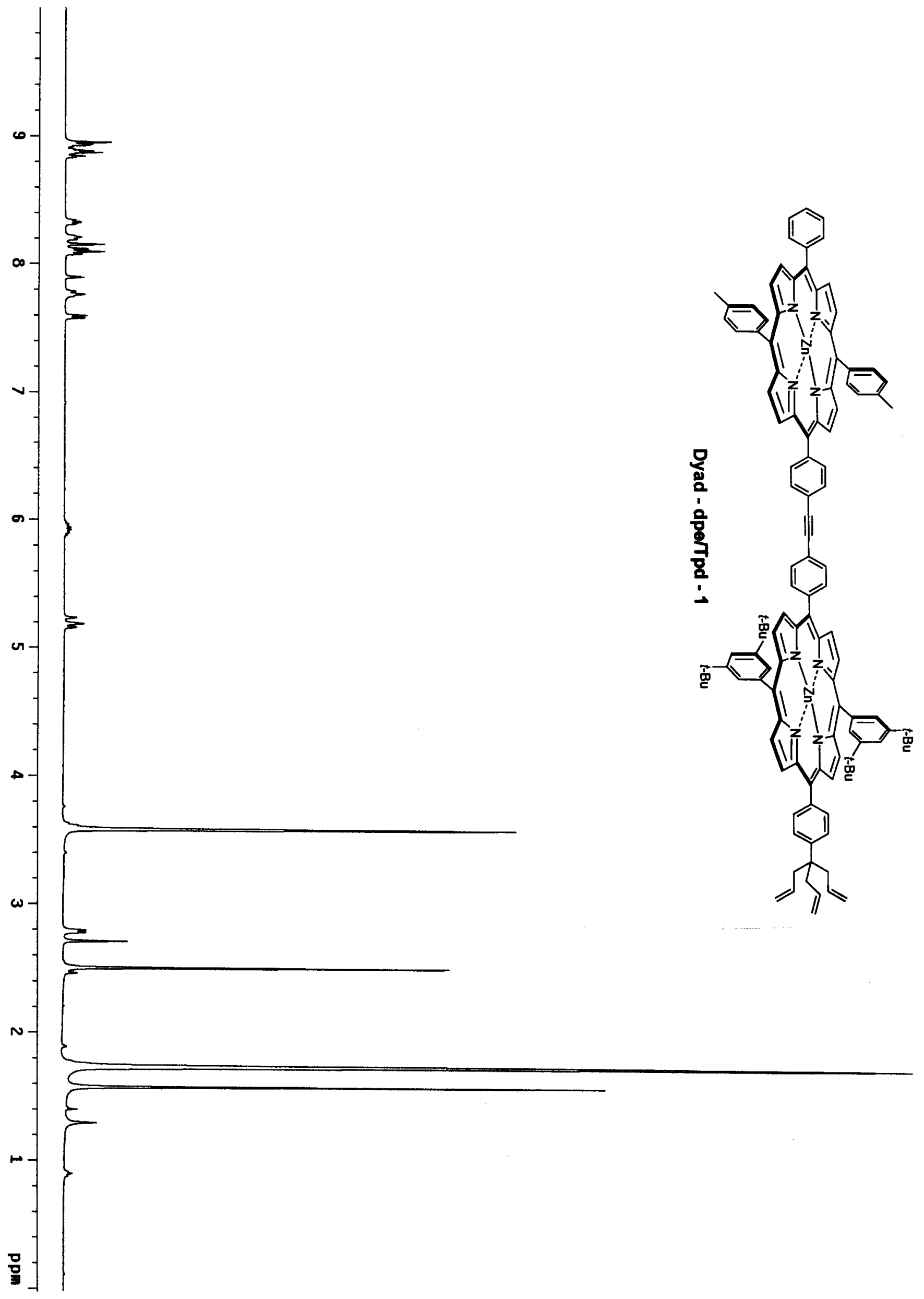




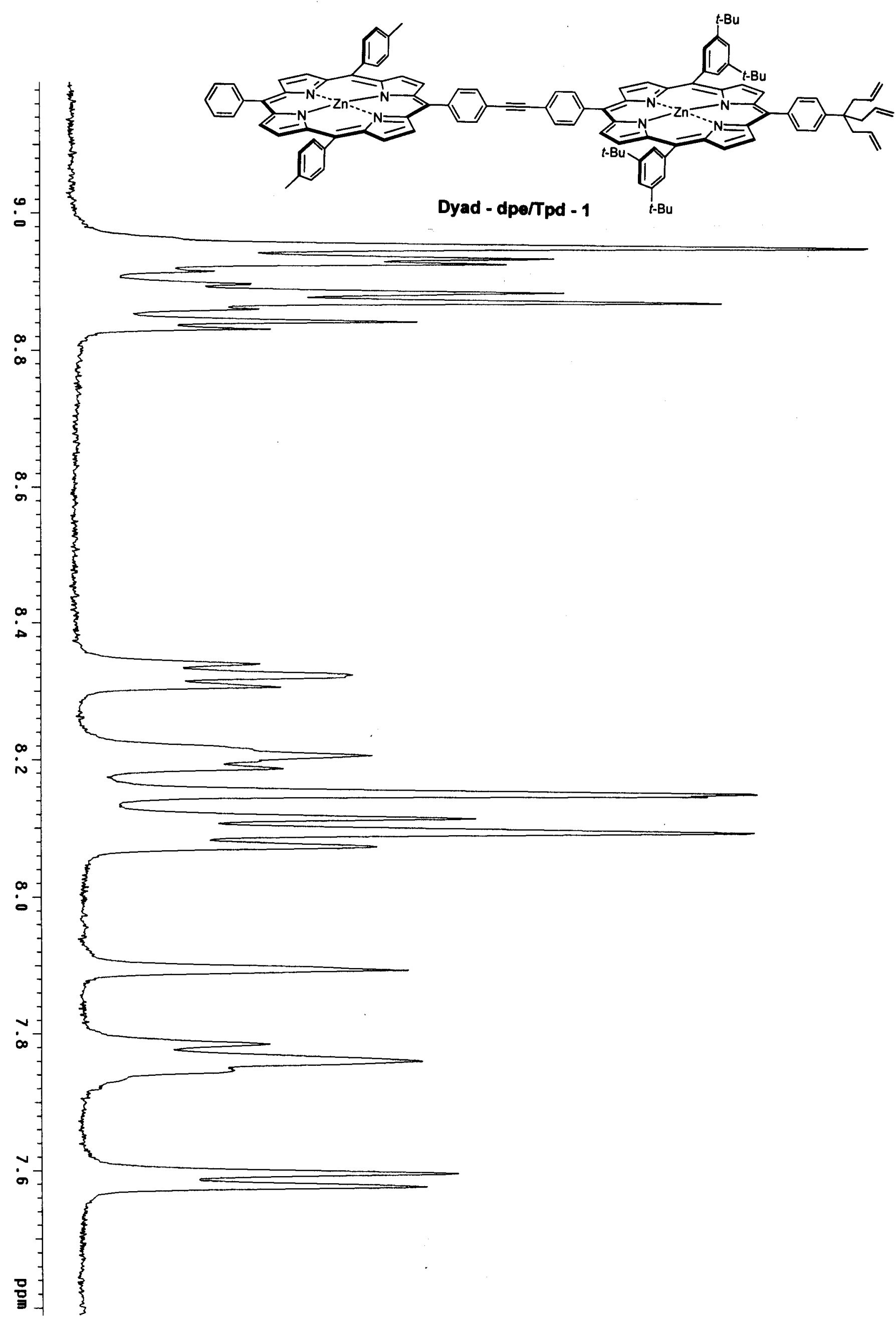




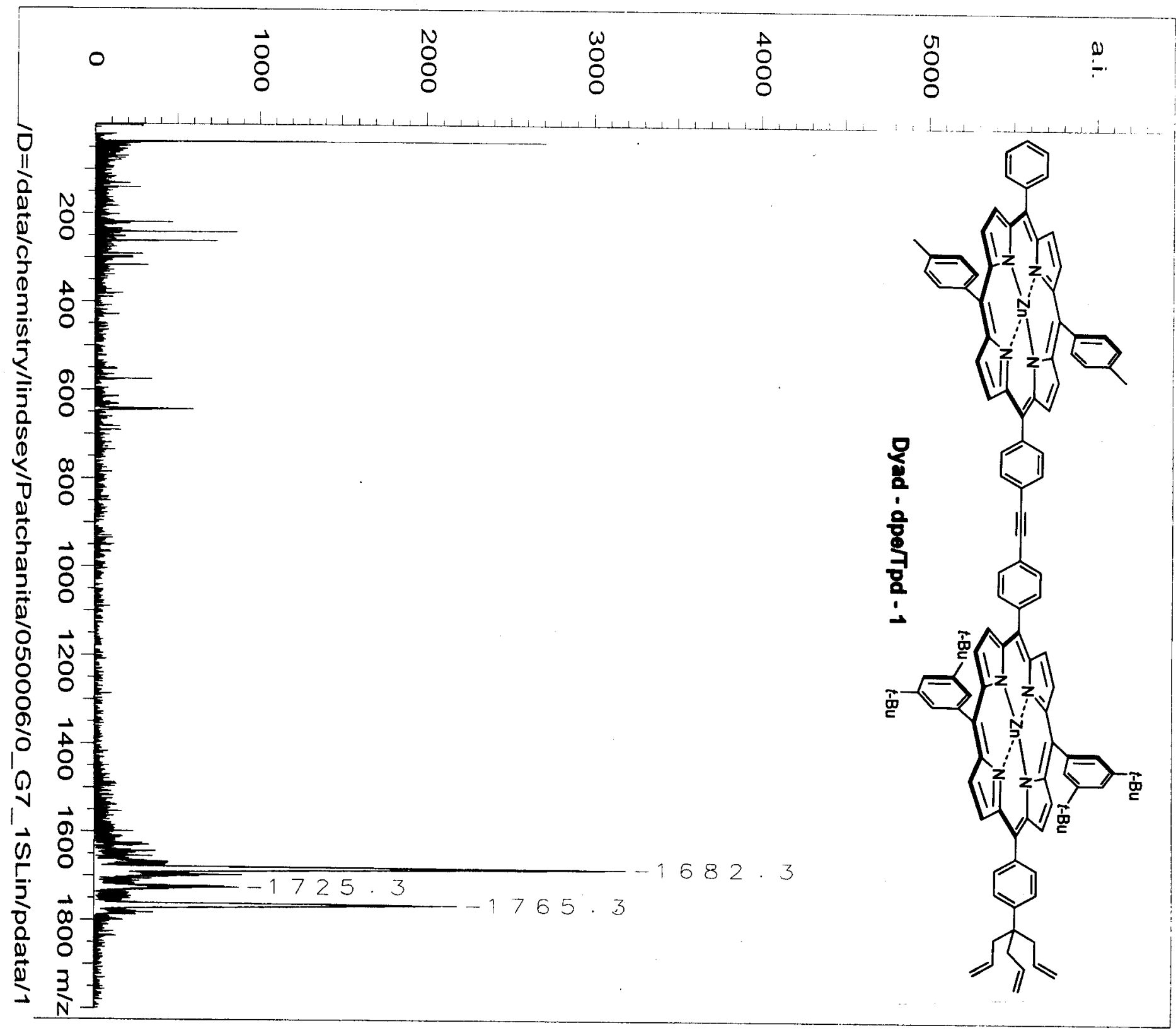

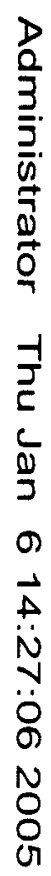

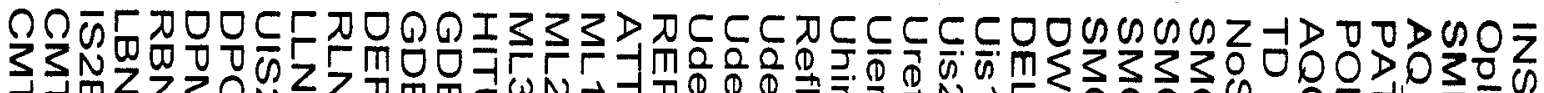

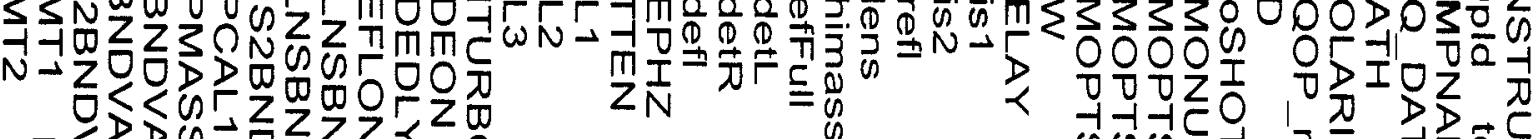

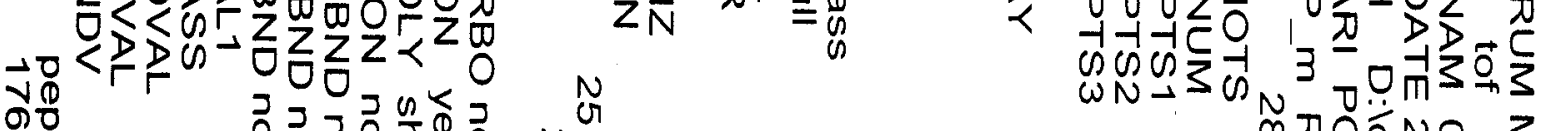

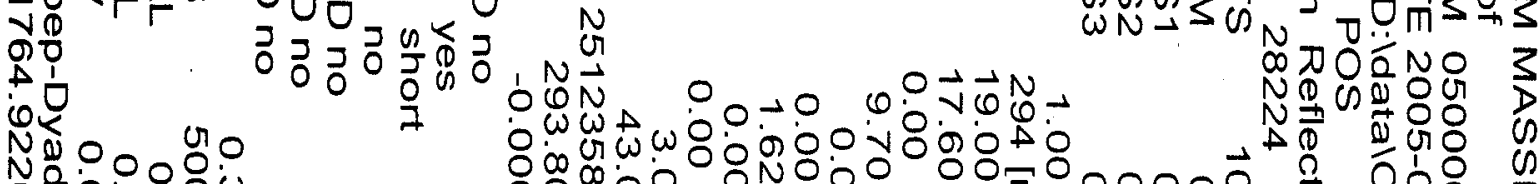
1.:०

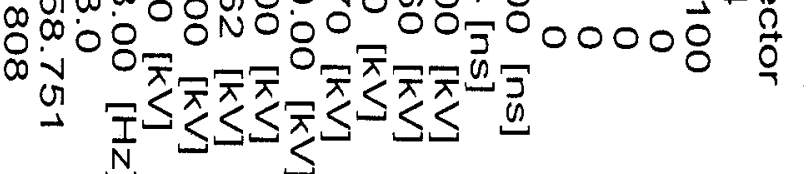

ฮี

亨

$\leq \vec{i}$ 


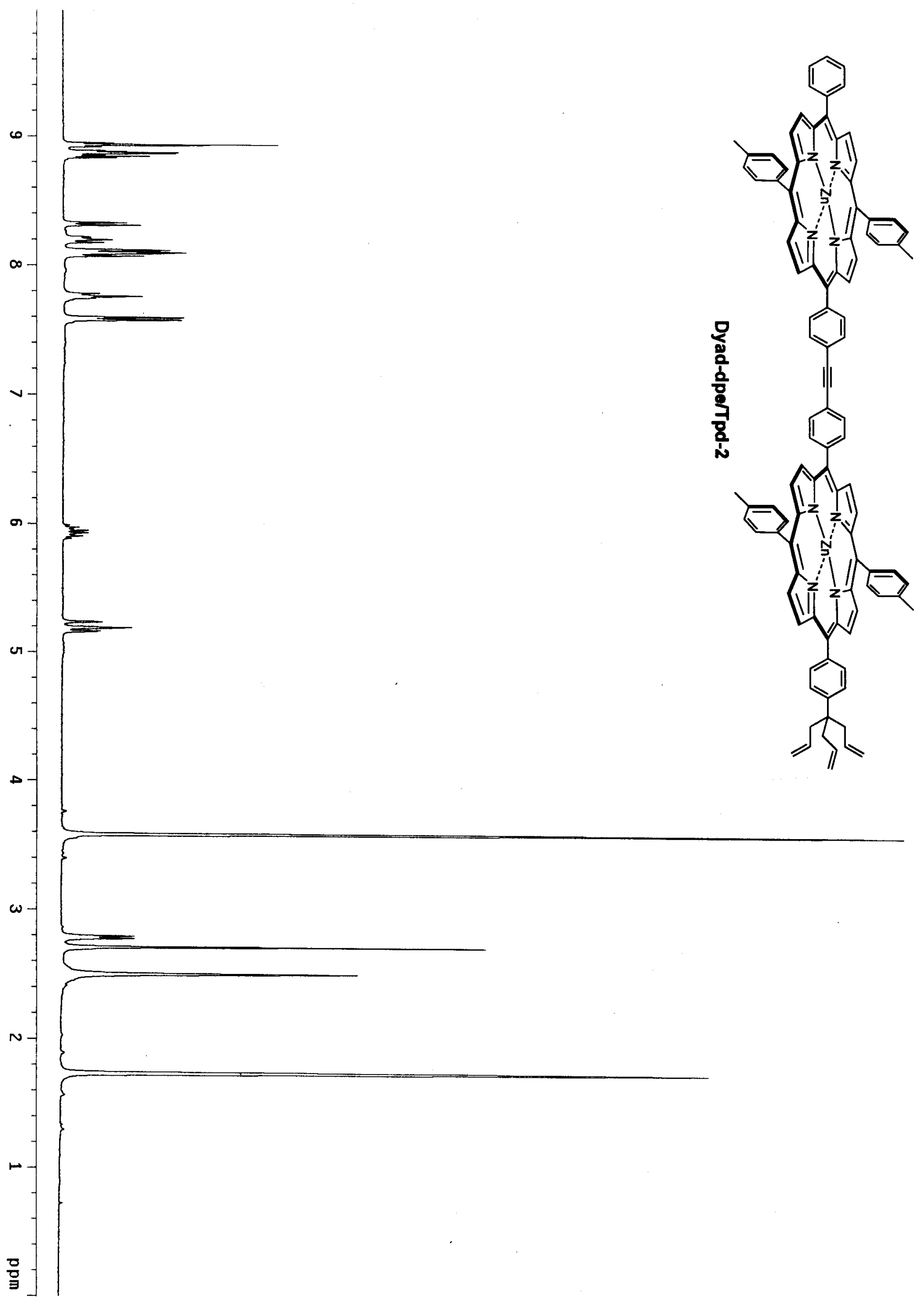




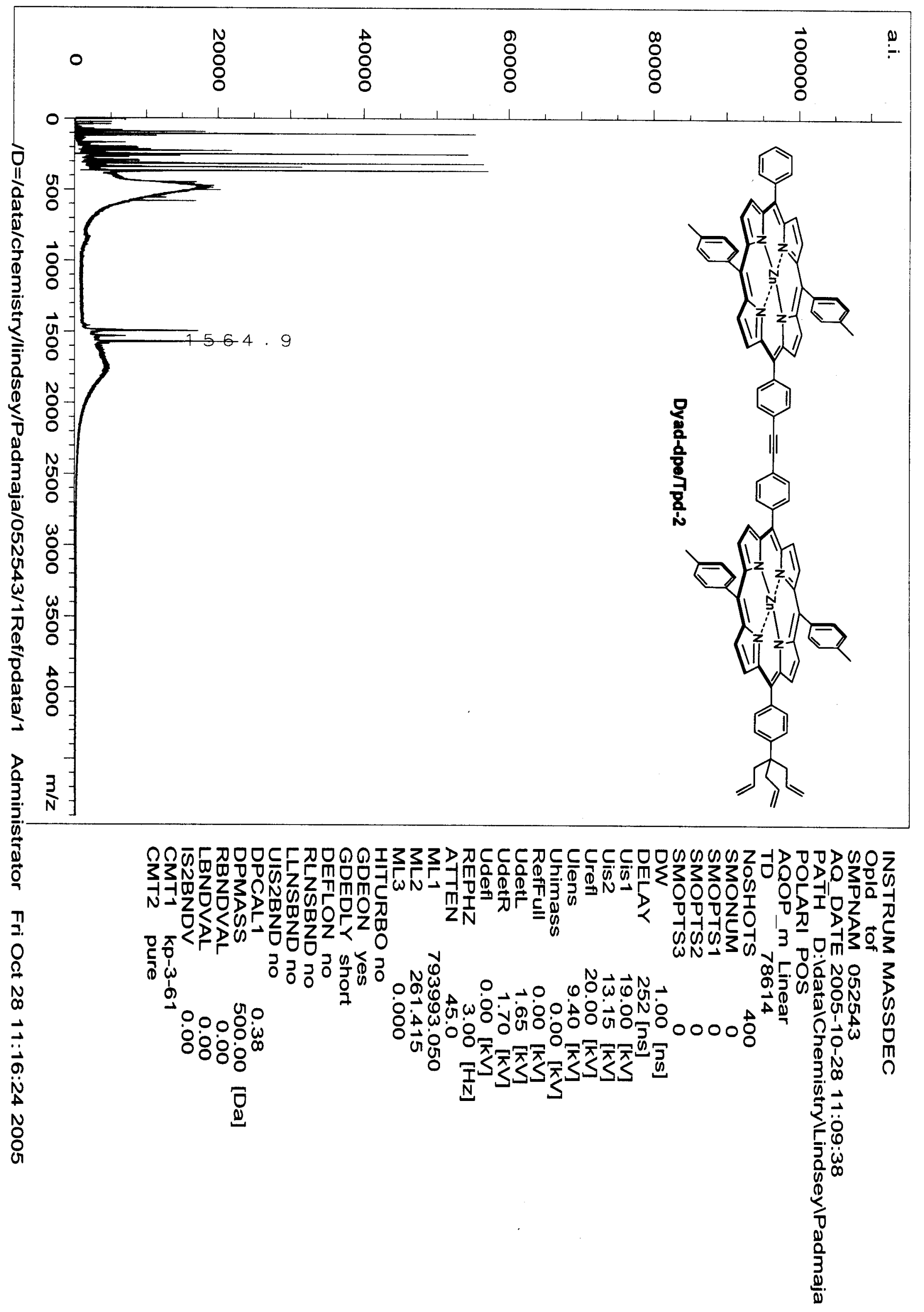




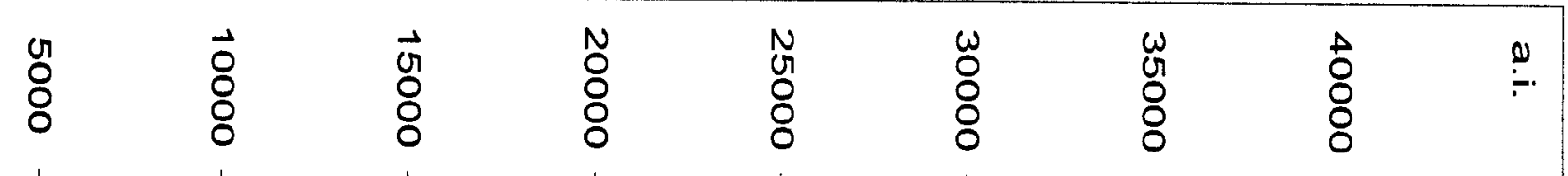

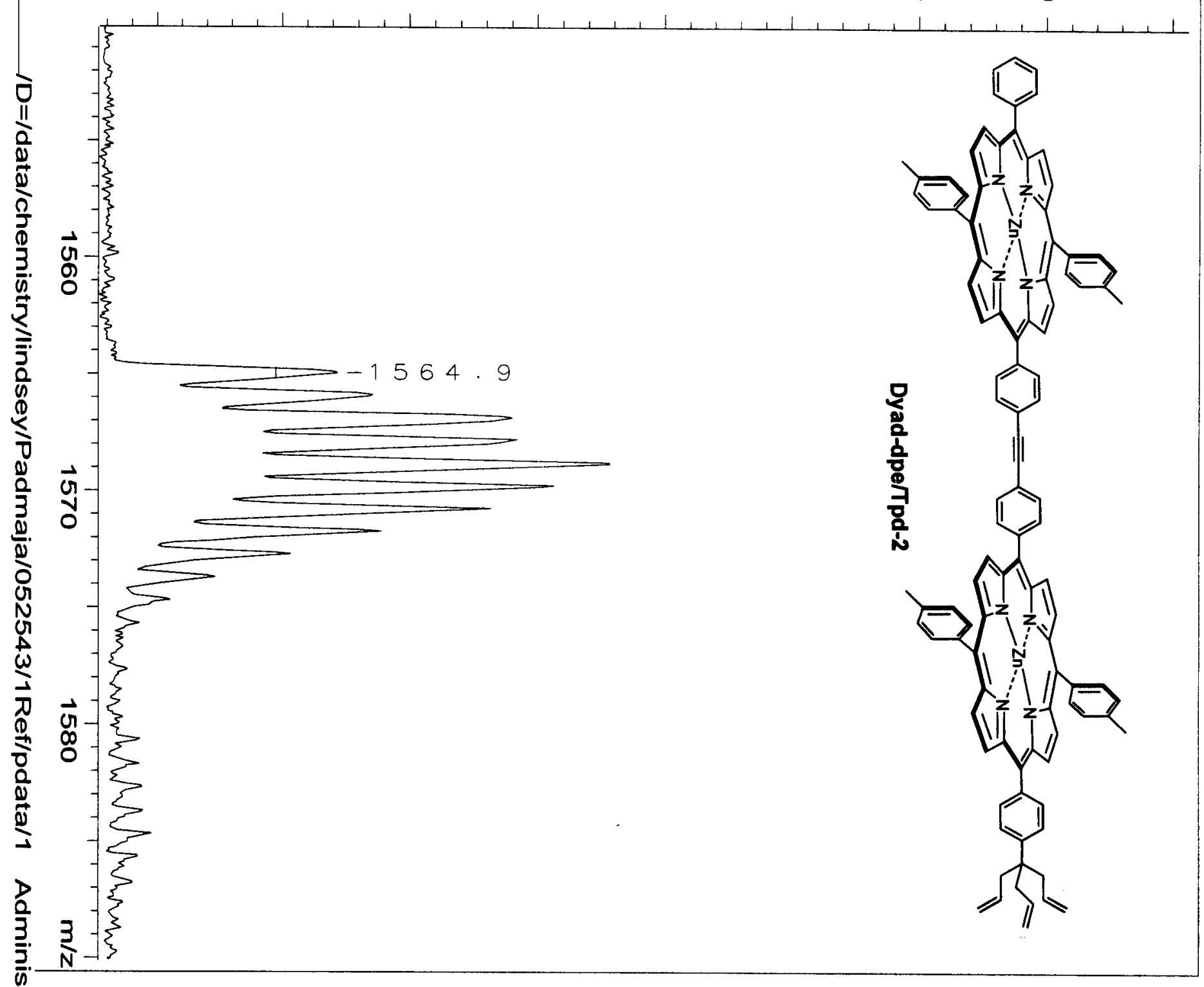

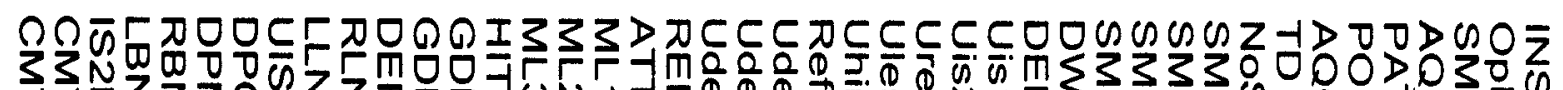
З3N

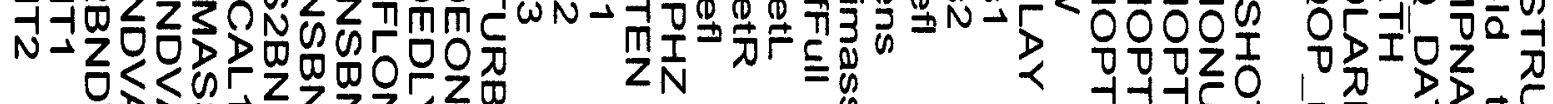

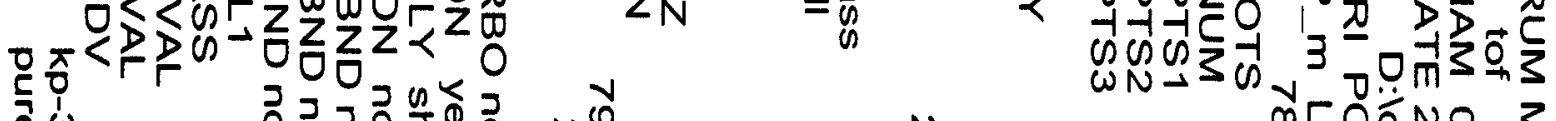

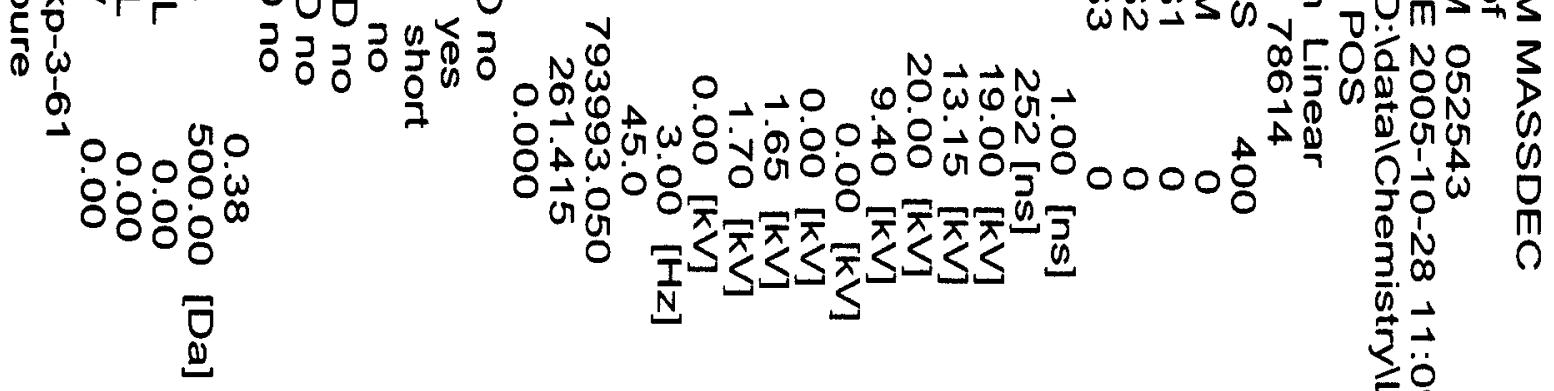

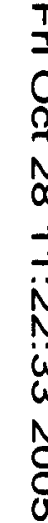

N 

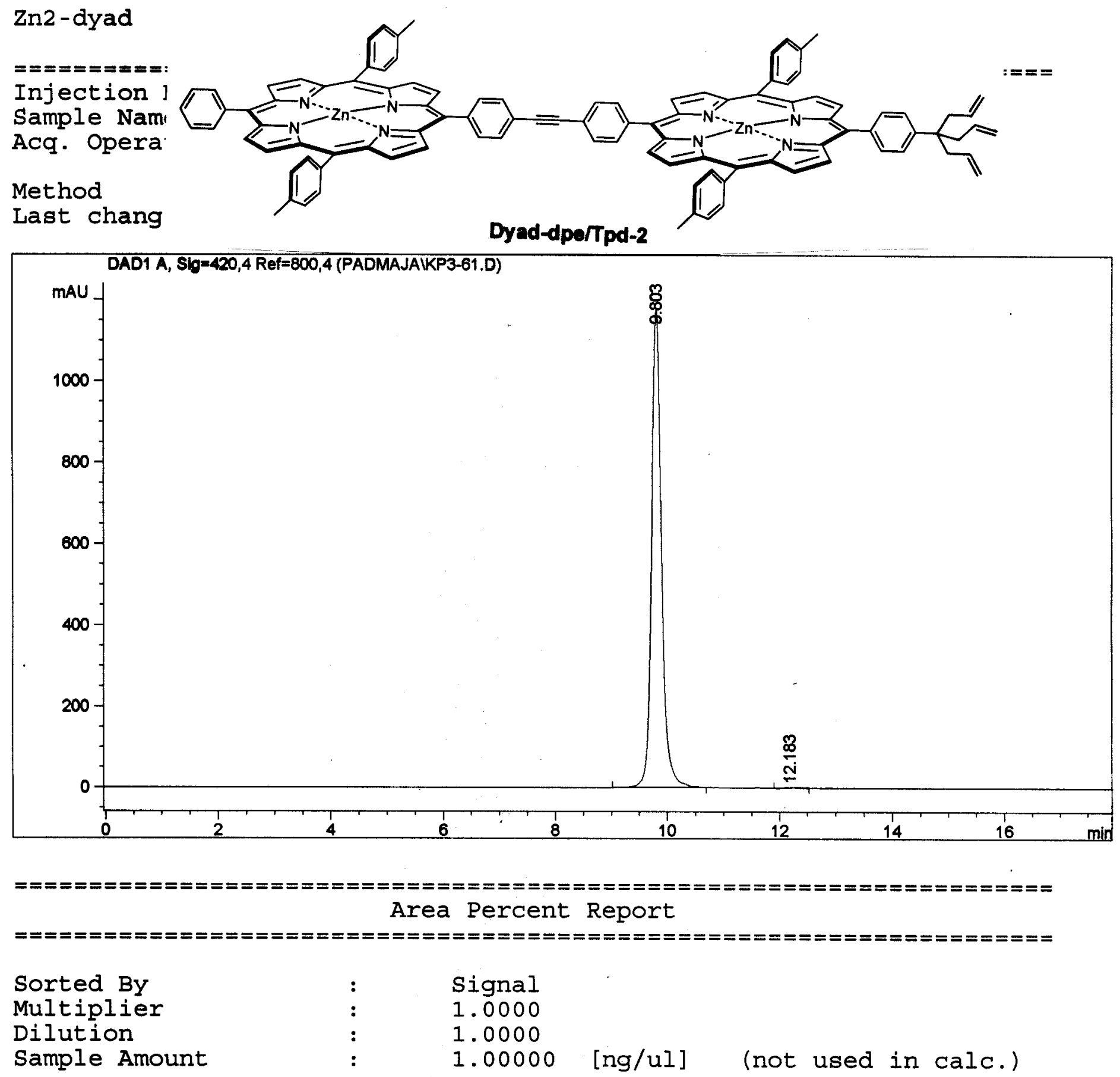

Signal 1: DAD1 A, Sig=420,4 Ref $=800,4$

\begin{tabular}{|c|c|c|c|c|c|c|}
\hline \# & $\begin{array}{l}\text { RetTime } \\
\text { [min] }\end{array}$ & Type & $\begin{array}{l}\text { Width } \\
\text { [min] }\end{array}$ & $\begin{array}{c}\text { Area } \\
{\left[\mathrm{mAU}{ }^{*} \mathrm{~g}\right]}\end{array}$ & $\begin{array}{l}\text { Height } \\
\text { [mAU] }\end{array}$ & $\begin{array}{c}\text { Area } \\
\frac{\circ}{\sigma}\end{array}$ \\
\hline $\begin{array}{l}1 \\
2\end{array}$ & $\begin{array}{r}9.803 \\
12.183\end{array}$ & $\begin{array}{l}\text { BB } \\
\text { PB }\end{array}$ & $\begin{array}{l}0 .: \\
0.7\end{array}$ & $\begin{array}{r}1.45526 \mathrm{e} 4 \\
16.07683\end{array}$ & $\begin{array}{r}1181.67432 \\
1.18526\end{array}$ & $\begin{array}{r}99.8896 \\
0.1104\end{array}$ \\
\hline
\end{tabular}

Totals : $\quad 1.45686 e 4 \quad 1182.85957$

Results obtained with enhanced integrator!

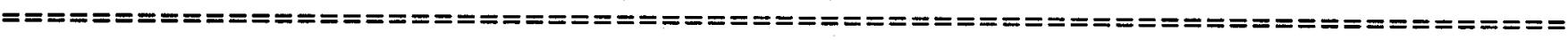
$\star \star \star$ End of Report $* \star *$ 
Print of window 38 : Current Chromatogram(s)

Current Chromatogram (s)

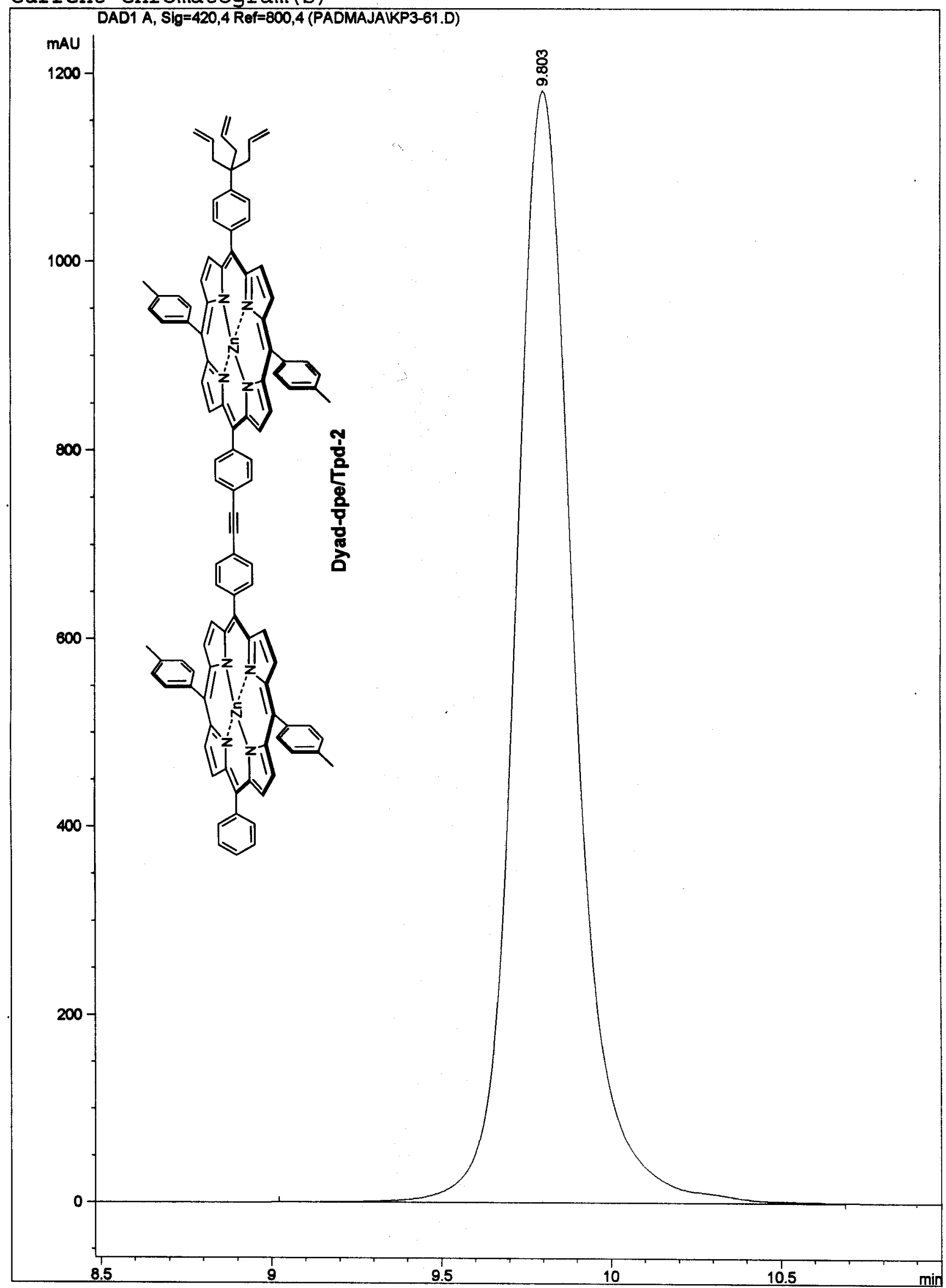

